\title{
Optimization of current carrying multicables
}

\author{
Helmut Harbrecht • Florian Loos
}

Received: date / Accepted: date

\begin{abstract}
Intense electric currents in cable bundles contribute to hotspot generation and overheating of essential car elements, especially in connecting structures. An important aspect in this context is the influence of the positioning of wires in cable harnesses. In order to find an appropriate multicable layout with minimized maximum temperatures, we formulate an optimization problem. Depending on the packing density of the cable bundle, it is solved via different optimization strategies: in case of loosely packed cable bundles solely by a gradient based strategy (shape optimization), densely packed ones by arrangement heuristics combined with a standard genetic algorithm, others by mixed strategies.

In the simulation model, the temperature dependence of electric resistances and different parameter values for the multitude of subdomains are respected. Convective and radiative effects are summarized by a heat transfer coefficient in a nonlinear boundary condition. Finite elements in combination with an interior-point method and a genetic algorithm allow the solution of the optimization problem for a large number of cable bundle types. Furthermore, we present an adjoint method for the solution of the shape optimization problem. The jumps at the interfaces of different materials are essential for the Hadamard representation of the shape gradient. Numerical experiments are carried out to demonstrate the feasibility and scope of the present approach.
\end{abstract}

\footnotetext{
This research has been supported in parts by the German National Science Foundation (DFG) through the Priority Program 1253 "Optimierung mit partiellen Differentialgleichungen" (Optimization with partial differential equations).

H. Harbrecht

Mathematisches Institut, Universität Basel, Rheinsprung 21, 4051 Basel, Switzerland

E-mail: helmut.harbrecht@unibas.ch

F. Loos

Institut für Mathematik und Bauinformatik, Universität der Bundeswehr München, WernerHeisenberg-Weg 39, 85577 Neubiberg, Germany

E-mail: florian.loos@unibw.de
} 
Keywords Shape optimization · Genetic algorithm · Electric cables · Finite element method · Joule heating

Mathematics Subject Classification (2000) 49Q10 $49 \mathrm{~J} 20 \cdot 65 \mathrm{~K} 10$

\section{Introduction}

The number of electric devices in modern cars grows continuously, whereas available space remains the same or even reduces. Especially in hybrid and electric cars, many components have to be supplied by intense currents. To save material, space and weight, manufacturers reduce cable diameters, which is only possible to a certain extent. Smaller diameters result in higher temperatures in the connecting structures. This could entail overheating and irreparable damages in essential components.

In [20], the modeling and simulation of heat transfer in current carrying multicables is described. Furthermore, the dependence of the heat distribution in the multicable on the composition and configuration of the single cables is shown by numerical experiments and measurements. Fig. 1 illustrates two different multicables, consisting of 33 single cables with same current and cross sectional area for each single cable in both cases. Furthermore, the multicable diameters, ambient temperatures and all other parameters are equal. Both only differ in the positioning of the single cables in the multicable. Obviously, the maximum temperature for the left single cable design is with $98.8^{\circ} \mathrm{C}$ essentially lower than for the right one with $107.4^{\circ} \mathrm{C}$.

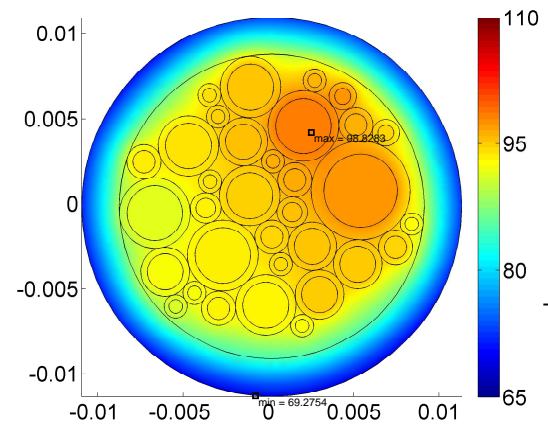

(a) Cable layout 1

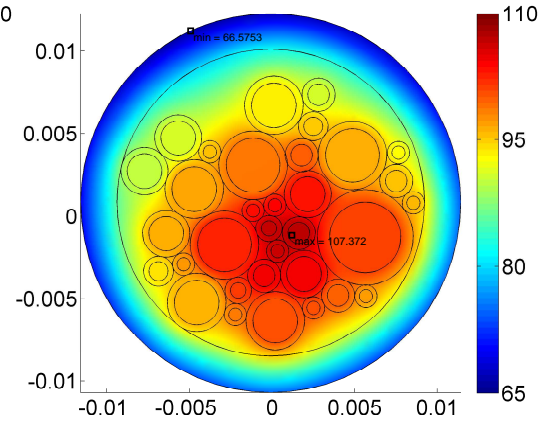

(b) Cable layout 2.

Fig. 1: Comparison of temperature distributions for equally composed multicables with different positions of the single cables.

In this paper, an algorithm to find an optimal multicable layout is developed. The electric current and the diameters of each single cable are fixed a 
priori. Dependent on the number of single cables and available space, different strategies are recommended. Whereas the exclusive application of gradient based shape optimization is sufficient in case of rather loosely packed cables, we additionally have to couple the gradient based strategy to a genetic algorithm for densely packed ones. For the shape optimization, we derive an adjoint system and the shape gradient as well as the local shape derivative. In addition, we present our genetic algorithm adapted to the problem.

The simulation of the temperature distribution in cable bundles and its computation is subject of several articles, e.g. $[3,4,18,20]$. In [2], algorithms for minimization of the total weight of cable bundles by given maximum temperature were developed. Therein, parallel multilevel methods are applied to heuristic strategies based on greedy type search methods. Our optimization problem is related. In contrast, we couple a non-gradient based strategy, namely a genetic algorithm, to a gradient based shape optimization approach. For introduction to the shape calculus and mathematical background, we recommend $[6,23,27]$. The particularity in the Hadamard representation of our shape gradient is due to the jumps in the coefficients of different materials at the interfaces. In $[11,12,15]$, methods to derive corresponding shape gradients are explained.

The paper is organized as follows: In Section 2, we introduce our modeling and simulation approach to describe the heating of multicables. Besides the state system, we formulate the optimal multicable layout problem. General information about shape optimization and genetic algorithms are explained in Section 3. In order to obtain an optimality system, we use the local shape derivative and derive an adjoint system as well as the shape gradient in Section 4. Section 5 describes numerical and computational details, including our optimization strategy combining heuristics for good initial positions, a squeezing algorithm, the numerical computation of the shape functional and the gradients, as well as the concrete implementation of the genetic algorithm. Numerical experiments are carried out in Section 6 and finally, we draw a conclusion in Section 7.

\section{Setting of the problem}

In this section, we introduce the mathematical notation, governing equations and formulate the optimal multicable layout problem.

\subsection{Notation}

Throughout this paper, we restrict ourselves to the stationary, two dimensional case. All vector valued functions are indicated by bold letters, e. g. the space variable $\mathbf{x}=(x, y)^{T} \in \mathbb{R}^{2}$ with norm $|\mathbf{x}|_{\mathbb{R}^{2}}=\left(x^{2}+y^{2}\right)^{1 / 2}$ and inner product $\left(\mathbf{x}_{1}, \mathbf{x}_{2}\right)$. The temperature in the multicable domain $\Omega^{\mathrm{MC}} \subset \mathbb{R}^{2}$ is dependent on the position $\mathbf{x}$ and denoted by $T=T(\mathbf{x})$. We abbreviate its gradient by 
$\nabla T=\left(\frac{\partial T}{\partial x}, \frac{\partial T}{\partial y}\right)^{T}$ and the divergence operator by $\nabla \cdot=\left(\left(\frac{\partial}{\partial x}, \frac{\partial}{\partial y}\right)^{T}, \cdot\right)$. The operator Div denotes the tangential divergence of a tangential field and $\nabla_{\tau}$ the tangential (surface) gradient of a scalar valued function.

Moreover, we identify $\mathbf{n}=\mathbf{n}_{\mathrm{e}}$ with the unit outer normal that points outward of the considered object, $\mathbf{n}_{\mathrm{i}}$ the unit inner normal pointing into the object. Consequently, the operator $\frac{\partial}{\partial \mathbf{n}}$ corresponds to the normal derivative of a quantity in outer direction. The notation $[\cdot]_{ \pm}$abbreviates the difference of the traces of a function at an interface boundary, approaching the boundary from exterior and interior respectively.

The $k$-th single cable $C_{k}=\left(x_{k}, y_{k}, r_{k}^{\mathrm{in}}, r_{k}^{\mathrm{ex}}\right)$ is geometrically described by the coordinates $\left(x_{k}, y_{k}\right)$ of its centre, the radius $r_{k}^{\text {in }}$ of the current carrying part and the outer radius $r_{k}^{\mathrm{ex}}$. The multicable $M C=\left(\left(x_{0}, y_{0}, r_{0}^{\mathrm{in}}, r_{0}^{\mathrm{ex}}\right) ; c_{1}, \ldots, c_{N}\right)$ consists of $N$ single cables (cf. Fig. 2a with $N=3$ ), has the centre coordinates $\left(x_{0}, y_{0}\right)$ and the inner radius $r_{0}^{\text {in }}$ respectively the outer radius $r_{0}^{\text {ex }}$. It is surrounded by an insulation layer of thickness $r_{0}^{\text {ex }}-r_{0}^{\text {in }}$ with constant heat conductivity $\lambda^{\mathrm{ex}}>0$. Each single cable consists of a core part $\Omega_{k}^{\text {core }}$ with constant heat conductivity $\lambda_{k}^{\text {core }}>0$, carrying the constant current $I_{k}$, and an insulation part $\Omega_{k}^{\text {iso }}$ with constant heat conductivity $\lambda_{k}^{\text {iso }}>0$. The gaps between the single cables and the exterior insulation can be of solid material or air. Herein, they are modelled by pure conduction with constant heat conductivity $\lambda^{\text {gaps }}$.

The interfaces between core and insulation part of each single cable and between single cable insulation and surrounding gaps play an important role in the calculation of the shape gradient. Thus, we introduce the interface boundaries $\Gamma_{k}^{\mathrm{i}}$ and $\Gamma_{k}^{\mathrm{e}}$ for $k=1, \ldots, N$ (cf. Fig. 2b with corresponding normal vectors on the boundaries).

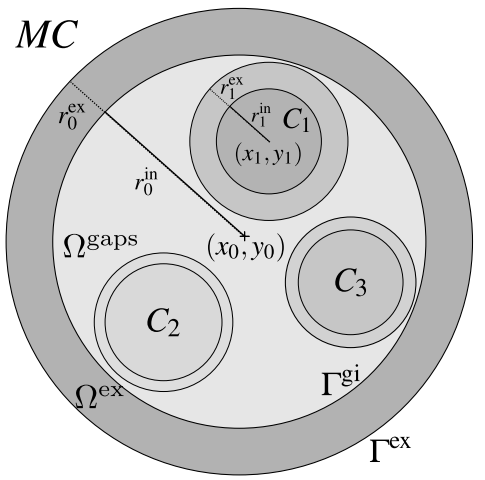

(a)

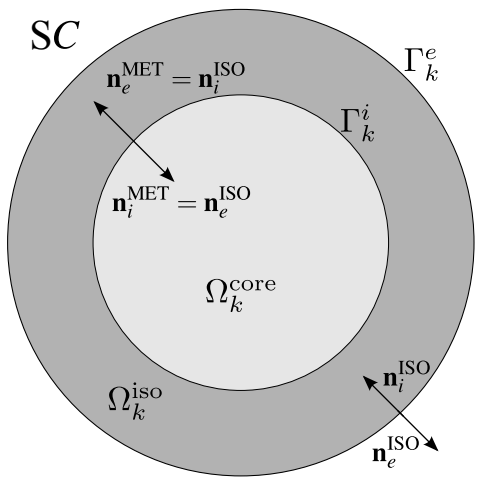

(b)

Fig. 2: Model of a multicable consisting of three single cables (a) and the $k$-th single cable with corresponding geometrical quantities (b). 
Furthermore, we introduce the set $\Gamma^{\mathrm{gi}}$ of interface boundaries between the exterior insulation and the (air) gaps (cf. Fig. 2a). All interface boundaries are summarized by $\Gamma^{\mathrm{int}}=\bigcup_{k=1}^{N}\left(\Gamma_{k}^{\mathrm{i}} \cup \Gamma_{k}^{\mathrm{e}}\right) \cup \Gamma^{\mathrm{gi}}$.

\subsection{State system}

Let the two dimensional cross sectional area of the multicable $\Omega^{\mathrm{MC}}$ be a connected bounded domain in $\mathbb{R}^{2}$ with regular exterior boundary $\partial \Omega^{\mathrm{MC}}=\Gamma^{\mathrm{ex}}$ and interface boundaries $\Gamma^{\text {int }}$. We suppose the cable to be entirely surrounded by air. Then, the continuous temperature distribution $T(\mathbf{x}) \in C\left(\Omega^{\mathrm{MC}}\right)$ is described by the state system which can be written in form of the following interface problem (cf. [20]):

$$
\begin{array}{rlrl}
-\nabla \cdot(\lambda \nabla T)-c \cdot T & =f & \text { in } \Omega^{\mathrm{MC}} \backslash \Gamma^{\mathrm{int}}, \\
\lambda^{\mathrm{ex}} \frac{\partial T}{\partial \mathbf{n}}+\alpha(T) \cdot\left(T-T^{\mathrm{amb}}\right) & =0 & & \text { on } \Gamma^{\mathrm{ex}}, \\
{[T]_{ \pm}=0 \quad \text { and } \quad\left[\lambda \frac{\partial T}{\partial \mathbf{n}}\right]_{ \pm}=0} & & \text { on } \gamma \in \Gamma^{\mathrm{int}},
\end{array}
$$

with

$$
\begin{aligned}
\lambda & :=\sum_{k=1}^{N}\left(\lambda_{k}^{\text {core }} \mathbb{I}_{\Omega_{k}^{\text {core }}}+\lambda_{k}^{\text {iso }} \mathbb{I}_{\Omega_{k}^{\text {iso }}}\right)+\lambda^{\text {ex }} \mathbb{I}_{\Omega^{\text {ex }}}+\lambda^{\text {gaps }} \mathbb{I}_{\Omega^{\text {gaps }}} \\
c & :=\sum_{k=1}^{N} \frac{1}{n_{k}}\left(\frac{4 I_{k}}{d_{k}^{\text {in }} \delta_{k} \pi}\right)^{2} \rho_{0, k} \alpha_{\rho, k} \mathbb{I}_{\Omega_{k}^{\text {core }}} \\
f & :=\sum_{k=1}^{N} \frac{1}{n_{k}}\left(\frac{4 I_{k}}{d_{k}^{\text {in }} \delta_{k} \pi}\right)^{2} \rho_{0, k}\left(1-\alpha_{\rho, k} T_{\text {ref }}\right) \mathbb{I}_{\Omega_{k}^{\text {core }}}
\end{aligned}
$$

Herein, the parameter $\lambda$ denotes the heat conductivity, $c$ the linear temperature coefficient and $f$ the source term. All are piecewise constant and vary only for different subdomains, expressed by the indicator function $\mathbb{I}$. Furthermore, the notation $\alpha(T)$ summarizes the heat transfer coefficient at the exterior boundary $\Gamma^{\mathrm{ex}}$, that represents the transition of the insulation material of the multicable to ambient air with temperature $T^{\mathrm{amb}}$. It is a smooth function in the range of temperatures $T$ under consideration and includes the effects of radiation and convection. The radiative part is governed by the StefanBoltzmann law [17], where $T$ appears in the power of three, the convective part is approximated by nonlinear formulas, obtained by fitting of empirical data $[1,18]$. For further details and concrete formulas to determine the convective part, we refer to [19], and for the identification of all other quantities to $[20]$.

Due to the jumps of the heat conductivity, the temperature profile is continuous across the interface of different materials $\gamma \in \Gamma^{\text {int }}$, but has kinks. 
Although the mentioning of these conditions is not necessary for a concise problem presentation, we indicate them explicitly in (1) to emphasize that it is an interface resp. transmission problem. The interface conditions are especially important for the Hadamard representation of the shape gradient.

Note finally that the governing equation of (1) is the Helmholtz equation. In the given form, it has in general no unique solution and oscillations can exist, especially for higher values of $c$. [10,20] give conditions for which the solution is still unique. It is proved that in the present application, the values of $c$ are small enough to provide a unique solution.

\subsection{Optimization problem}

Let the dimensions of the exterior insulation and the single cables be given. The material parameters and the current of each single cable are fixed and known, such that $\lambda, c$ and $f$ are determined a priori. What we vary is the positioning of the single cables, summarized by the domain

$$
\Omega:=\bigcup_{k=1}^{N} \Omega_{k}=\bigcup_{k=1}^{N}\left(\Omega_{k}^{\text {core }} \cup \Omega_{k}^{\text {iso }}\right),
$$

with $\Omega_{k}$ the domain of single cable $k$. As the position of each single cable is determined by its centre, our optimization variables are the centre coordinates of the single cables $\left(x_{1}, y_{1}\right), \ldots,\left(x_{N}, y_{N}\right)$. Consequently, the optimal multicable layout problem (OptMC) with varying domain $\Omega$ reads as

$$
\begin{gathered}
J(\Omega)=\sum_{k=1}^{N} \int_{\Omega_{k}} j(\mathbf{x}, T(\mathbf{x})) \mathrm{d} \mathbf{x}+\int_{\Omega^{\mathrm{MC}} \backslash \cup_{k=1}^{N} \Omega_{k}} j(\mathbf{x}, T(\mathbf{x})) \mathrm{d} \mathbf{x} \rightarrow \min ! \\
\text { where }-\nabla \cdot(\lambda \nabla T)-c \cdot T=f \quad \text { in } \Omega^{\mathrm{MC}} \backslash \Gamma^{\mathrm{int}}, \\
\lambda^{\mathrm{ex}} \frac{\partial T}{\partial \mathbf{n}}+\alpha(T) \cdot\left(T-T^{\mathrm{amb}}\right)=0 \quad \text { on } \Gamma^{\mathrm{ex}}, \\
{[T]_{ \pm}=0 \quad \text { and } \quad\left[\lambda \frac{\partial T}{\partial \mathbf{n}}\right]_{ \pm}=0 \quad \text { on } \gamma \in \Gamma^{\mathrm{int}},} \\
\text { s.t. }\left(x_{k}-x_{0}\right)^{2}+\left(y_{k}-y_{0}\right)^{2}<\left(r_{k}^{\mathrm{ex}}-r_{0}^{\mathrm{in}}\right)^{2}, \quad k \in K=\{1, \ldots, N\}, \\
\left(x_{k}-x_{l}\right)^{2}+\left(y_{k}-y_{l}\right)^{2}>\left(r_{k}^{\mathrm{ex}}+r_{l}^{\mathrm{ex}}\right)^{2}, \quad(k, l) \in(K \times K) \backslash\{k=l\}, \\
x_{1}=0, \quad 0<y_{1}<r_{0}^{\mathrm{in}}, \quad 0<x_{2}<r_{0}^{\mathrm{in} .}
\end{gathered}
$$

It is the objective to minimize the cost functional $J(\Omega)$ respectively the function $j(\mathbf{x}, T(\mathbf{x}))$ over the domain $\Omega^{\mathrm{MC}}$ subject to the state system (1) and geometrical constraints. To that end, we shall assume that $j \in C^{1}\left(\bar{\Omega}^{\mathrm{MC}} \times \mathbb{R}_{\geq 0}\right)$. We furthermore require that each single cable has to be entirely inside the 
multicable and single cables must not overlap. ${ }^{1}$ This problem is rotationally symmetrical because we apply the same boundary condition to the entire exterior boundary. Thus, we allow the first cable to be positioned only in the vertical upper line segment connecting the multicable centre and the point $\left(0, r_{0}^{\text {in }}\right)$. To avoid axial symmetry, the second cable has to be in the right sector of the multicable.

Note once more that the domain $\Omega$ of the single cables is composed of the subdomains $\Omega_{k}, k=1, \ldots, N$, which themselves depend on the subdomains $\Omega_{k}^{\text {core }}$ and $\Omega_{k}^{\text {iso }}$. With $d(\cdot, \cdot)$ the distance between two points, we introduce the notation

$$
B_{r}(\mathbf{c})=\left\{\mathbf{x} \in \mathbb{R}^{2} \mid d(\mathbf{c}, \mathbf{x})<r\right\},
$$

describing the open ball of radius $r>0$, centred at a point $\mathbf{c} \in \mathbb{R}^{2}$. Thus, the core and the insulation of single cable $k$ are determined by

$$
\Omega_{k}^{\text {core }}=B_{r_{k}^{\text {in }}}\left(\left(x_{k}, y_{k}\right)^{T}\right), \Omega_{k}^{\text {iso }}=B_{r_{k}^{\text {ex }}}\left(\left(x_{k}, y_{k}\right)^{T}\right) \backslash \Omega_{k}^{\text {core }} .
$$

The dependence of the single cable $k$ on the coordinates $\left(x_{k}, y_{k}\right)$ is obvious and consequently the dependence of the entire domain $\Omega$ of the single cables on all centres, abbreviated by the notation

$$
\Omega=\Omega\left(\left(x_{1}, y_{1}\right), \ldots,\left(x_{N}, y_{N}\right)\right) .
$$

We summarize the set of admissible domains by

$$
\begin{aligned}
\mathcal{O}_{\mathrm{ad}}=\{ & \Omega=\Omega\left(\left(x_{1}, y_{1}\right), \ldots,\left(x_{N}, y_{N}\right)\right) \in \mathbb{R}^{2} \mid \\
& \left(x_{k}-x_{0}\right)^{2}+\left(y_{k}-y_{0}\right)^{2}<\left(r_{k}^{\mathrm{ex}}-r_{0}^{\mathrm{in}}\right)^{2}, k \in K=\{1, \ldots, N\}, \\
& \left(x_{k}-x_{l}\right)^{2}+\left(y_{k}-y_{l}\right)^{2}>\left(r_{k}^{\mathrm{ex}}+r_{l}^{\mathrm{ex}}\right)^{2},(k, l) \in(K \times K) \backslash\{k=l\}, \\
& \left.x_{1}=0,0<y_{1}<r_{0}^{\mathrm{in}}, 0<x_{2}<r_{0}^{\mathrm{in}}\right\} .
\end{aligned}
$$

The exterior insulation and thus the exterior boundary of the multicable domain $\Omega^{\mathrm{MC}}$ is fixed in this setting. In our optimization approach, we consider the multicable as a composition of several subdomains. Thus, the interface boundaries of $\Omega^{\mathrm{MC}}$ vary for different shapes of the domain $\Omega$ of the single cables, which shall be indicated by the notation $\Omega^{\mathrm{MC}}(\Omega)$.

Throughout this work, we assume that all interface boundaries $\Gamma_{k}^{\mathrm{i}}, \Gamma_{k}^{\mathrm{e}}, k=$ $1, \ldots, N, \Gamma^{\mathrm{gi}}$ and the exterior boundary $\Gamma^{\mathrm{ex}}$ are $C^{2}$-smooth.

\section{Optimization}

Our algorithm to optimize multicables combines gradient based shape optimization and a genetic algorithm. In this section, we provide general information about shape optimization and genetic algorithms.

\footnotetext{
1 To avoid problems in the calculation with finite elements, we require in the numerical implementation that the distance between each single cable and multicable insulation respectively pairwise between two single cables has to be larger than $\delta>0$. The parameter $\delta$ is chosen such that the space in between is sufficiently large to create a feasible mesh.
} 
3.1 Shape optimization

Shape calculus will be used to derive the shape gradient of the shape optimization problem (3) under considerations. For a general overview on shape calculus, mainly based on the perturbation of identity (Murat and Simon) or the speed method (Sokolowski and Zolesio), we refer the reader for example to $[6,22,23,27]$ and the references therein.

For a smooth perturbation field $\mathbf{V}: D \rightarrow \mathbb{R}^{n}$, we consider the perturbed domain

$$
\Omega_{\epsilon}[\mathbf{V}]:=\{\mathbf{x}+\epsilon \mathbf{V}(\mathbf{x}): \mathbf{x} \in \Omega\},
$$

with $\epsilon>0$ sufficiently small [22] (cf. Fig. 3 for an illustration). This enables the definition of the shape derivative of the shape functional $J$ at $\Omega$ in direction of a vector field $\mathbf{V}$ by

$$
\delta J(\Omega)[\mathbf{V}]:=\lim _{\epsilon \rightarrow 0} \frac{J\left(\Omega_{\epsilon}[\mathbf{V}]\right)-J(\Omega)}{\epsilon} .
$$

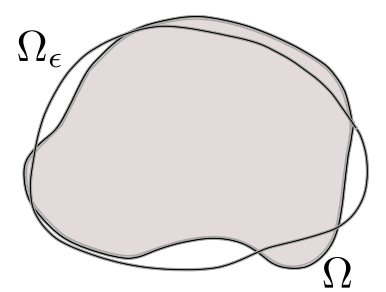

Fig. 3: An arbitrary domain $\Omega$ and a corresponding perturbed domain $\Omega_{\epsilon}$.

The shape functional $J$ is shape differentiable at $\Omega$, if the Eulerian derivative $\delta J(\Omega)[\mathbf{V}]$ exists for all directions $\mathbf{V}$ and if the mapping $\mathbf{V} \mapsto \delta J(\Omega)[\mathbf{V}]$ is linear and continuous. In particular, according to the Hadamard-Zolésio structure theorem $[6,26,27]$, it is known that the shape gradient $g$ can be expressed as a boundary integral of the form

$$
\delta J(\Omega)[\mathbf{V}]=\int_{\Gamma}\langle\mathbf{V}, \mathbf{n}\rangle g \mathrm{~d} \sigma
$$

where $\Gamma$ denotes the varying boundary or interface and $\langle\cdot, \cdot\rangle$ denotes the scalar product in $\mathbb{R}^{n}$.

Furthermore, we shall introduce the local shape derivative $\delta u=\delta u[\mathbf{V}]$ that describes the sensitivity of the PDE solution with respect to domain variations. It is defined pointwise by

$$
\delta u(\mathbf{x}):=\lim _{\epsilon \rightarrow 0} \frac{u_{\epsilon}(\mathbf{x})-u(\mathbf{x})}{\epsilon}, \quad \mathbf{x} \in \Omega \cap \Omega_{\epsilon},
$$


with the solution of the boundary value problem on the perturbed domain denoted by $u_{\epsilon}$.

The local shape derivative $\delta u=\delta u[\mathbf{V}]$ and the shape derivative $\delta J(\Omega)[\mathbf{V}]$ are essential for the sensitivity analysis of the problem in Section 4 .

\subsection{Genetic algorithm}

A certain class of optimization methods is summarized in the term genetic algorithm. We describe how a genetic algorithm generally works, based on [13, 14,21].

A genetic algorithm is a stochastic method that can be used to solve optimization problems, e.g. to find a function's minimum. However, it is not as precise as a gradient based method, as it does not study the function to be minimized. It only evaluates the function for a given number of optimization variable values (individuals).

The algorithm employs the concept of natural evolution (cf. Fig. 4): an initial population of individuals evolves in several generations, using the simulated genetic operations crossover and mutation, which let the fittest individuals survive and reproduce. The initial population is made up of the $M$ individuals, obtained e.g. via the specified heuristics, and can be completed by random individuals.

We evaluate the fitness of each individual (i. e. its objective function value) in order to determine which individuals are the 'better' ones. To simulate the transition from one generation to the next one, we use the following steps:

- The reproduction is subject to the fitness: the $k$ best individuals are directly transmitted to the next generation as elite and the parents are selected stochastically or with a certain strategy in the present population.

- Some parents are crossed and mutated to produce the 'children'.

- The new population replaces the old one.

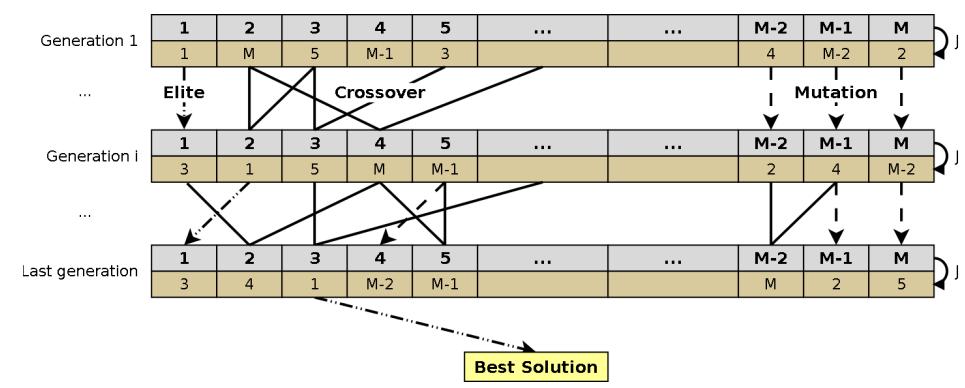

Fig. 4: Diagram of the general procedure of a genetic algorithm. 


\subsubsection{Crossover}

Crossover children are created by combining the vectors of a pair of parents: we randomly select components from both parents (here: $p_{1}$ and $p_{2}$ ) and create the child (here: $k$ ) with these components. For example, for two parents

$$
\begin{aligned}
& p_{1}=[1,2,3,4,5,6,7], \\
& p_{2}=[a, b, c, d, e, f, g],
\end{aligned}
$$

one child could be

$$
k=[1,2, c, 4, e, f, 7]
$$

\subsubsection{Mutation}

Mutation children are created by randomly modifying a certain number of components of the parent. We pick some components of the parent and take the average of these components with a random number $r_{i}$. For example, a mutation of

$$
p=[a, b, c, d, e, f, g] \quad \text { could be } \quad k=\left[a, \frac{b+r_{1}}{2}, c, d, \frac{e+r_{2}}{2}, \frac{f+r_{3}}{2}, g\right] \text {. }
$$

Each created individual (by crossover or mutation) has to be tested to ensure that it respects the constraints of the optimization problem. If however it does not, we have to modify it until it respects the given criteria, or if adaptation fails, the non-conform individual is erased and a new one is created.

\subsubsection{Stopping criterion}

Since there is no natural stopping criterion for this algorithm, it is interrupted in general either after a certain number of generations or if there is no improvement in the fitness of the best individual in the population for several steps.

\section{Sensitivity analysis}

In the following, we apply an adjoint method to compute the shape gradient. Thus, we derive an adjoint system, the Hadamard representation of the shape gradient and the local shape derivative of the problem. We suppose the underlying functions to be sufficiently smooth to ensure well-posedness of all operations. 
4.1 Local shape derivative

Before deriving the shape gradient, we state the system holding for the local shape derivative $\delta T[\mathbf{V}]$ of (OptMC). To this end, let $\mathbf{V} \in C^{2}\left(\bar{\Omega} ; \mathbb{R}^{2}\right)$ be a smooth domain variation of $\Omega$. Then, there holds:

$$
\begin{gathered}
\nabla \cdot(\lambda \nabla \delta T[\mathbf{V}])+c \cdot \delta T[\mathbf{V}]=0 \text { in } \Omega^{\mathrm{MC}} \backslash \Gamma^{\mathrm{int}} \\
\lambda^{\operatorname{ex}} \frac{\partial \delta T[\mathbf{V}]}{\partial \mathbf{n}}+\left(\alpha^{\prime}(T)\left(T-T^{\mathrm{amb}}\right)+\alpha(T)\right) \delta T[\mathbf{V}]=0 \text { on } \Gamma^{\mathrm{ex}}, \\
{\left[\lambda \frac{\partial \delta T[\mathbf{V}]}{\partial \mathbf{n}}\right]_{ \pm}=\operatorname{Div}\left(\langle\mathbf{V}, \mathbf{n}\rangle[\lambda]_{ \pm} \nabla_{\tau} T\right)+\left([c]_{ \pm} T+[f]_{ \pm}\right)\langle\mathbf{V}, \mathbf{n}\rangle} \\
\text { and }[\delta T[\mathbf{V}]]_{ \pm}=-\langle\mathbf{V}, \mathbf{n}\rangle\left[\frac{\partial T}{\partial \mathbf{n}}\right]_{ \pm} \text {on } \gamma \in \Gamma^{\mathrm{int}} .
\end{gathered}
$$

Proof The pointwise evaluation of the boundary value problem for $T$ respectively $T_{\epsilon}[\mathbf{V}]$ on the domains $\Omega^{\mathrm{MC}}(\Omega)$ respectively $\Omega^{\mathrm{MC}}\left(\Omega_{\epsilon}[\mathbf{V}]\right)$ in a point $\mathbf{x} \in \Omega^{\mathrm{MC}}$ provides

$$
\lim _{\epsilon \rightarrow 0} \frac{\nabla \cdot\left(\lambda \nabla T_{\epsilon}[\mathbf{V}]\right)+c \cdot T_{\epsilon}[\mathbf{V}]-(\nabla \cdot(\lambda \nabla T)+c \cdot T)}{\epsilon}=-f+f=0,
$$

i. e. the partial differential equation

$$
\nabla \cdot(\lambda \nabla \delta T[\mathbf{V}])+c \cdot \delta T[\mathbf{V}]=0 \text { in } \Omega^{\mathrm{MC}} \backslash \Gamma^{\mathrm{int}} .
$$

The condition on the exterior boundary is

$$
\lim _{\epsilon \rightarrow 0} \lambda^{\operatorname{ex}} \frac{\frac{\partial T_{\epsilon}[\mathbf{V}]}{\partial \mathbf{n}}-\frac{\partial T}{\partial \mathbf{n}}}{\epsilon}=-\lim _{\epsilon \rightarrow 0} \frac{\alpha\left(T_{\epsilon}[\mathbf{V}]\right)\left(T_{\epsilon}[\mathbf{V}]-T^{\mathrm{amb}}\right)-\alpha(T)\left(T-T^{\mathrm{amb}}\right)}{\epsilon} .
$$

Here, the term on the left hand side yields

$$
\lim _{\epsilon \rightarrow 0} \lambda^{\operatorname{ex}} \frac{\frac{\partial T_{\epsilon}[\mathbf{V}]}{\partial \mathbf{n}}-\frac{\partial T}{\partial \mathbf{n}}}{\epsilon}=\lambda^{\operatorname{ex}} \frac{\partial \delta T[\mathbf{V}]}{\partial \mathbf{n}},
$$

while the term on the right hand side provides

$$
\begin{aligned}
& \lim _{\epsilon \rightarrow 0} \frac{\alpha\left(T_{\epsilon}[\mathbf{V}]\right)\left(T_{\epsilon}[\mathbf{V}]-T^{\mathrm{amb}}\right)-\alpha(T)\left(T-T^{\mathrm{amb}}\right)}{\epsilon} \\
& =\lim _{\epsilon \rightarrow 0}\left(\frac{\alpha\left(T_{\epsilon}[\mathbf{V}]\right)-\alpha(T)}{\epsilon}\left(T_{\epsilon}[\mathbf{V}]-T^{\mathrm{amb}}\right)+\alpha(T) \frac{T_{\epsilon}[\mathbf{V}]-T}{\epsilon}\right) \\
& =\left(\alpha^{\prime}(T)\left(T-T^{\mathrm{amb}}\right)+\alpha(T)\right) \delta T[\mathbf{V}] .
\end{aligned}
$$

Putting the latter two identities together yields the desired boundary conditions at $\Gamma^{\mathrm{ex}}$.

Finally, for the interface conditions of the local shape derivative, we refer to $[15,16]$. 
4.2 Shape gradient

For an optimal interior point, the necessary optimality condition

$$
\delta J(\Omega)[\mathbf{V}]=0
$$

has to be fulfilled for all directions V. The Hadamard representation of the shape gradient for (OptMC) reads as follows:

$$
\begin{aligned}
& \delta J(\Omega)[\mathbf{V}]=\sum_{\gamma \in \Gamma^{\mathrm{int}}} \int_{\gamma}\langle\mathbf{V}, \mathbf{n}\rangle\left\{\nabla_{\tau} p^{\mathrm{e}} \nabla_{\tau} T^{\mathrm{e}}[\lambda]_{ \pm}\right. \\
&\left.\quad-p^{\mathrm{e}}\left([c]_{ \pm} T^{\mathrm{e}}+[f]_{ \pm}\right)-\lambda^{\mathrm{e}} \frac{\partial p^{\mathrm{e}}}{\partial \mathbf{n}}\left[\frac{\partial T}{\partial \mathbf{n}}\right]_{ \pm}\right\} \mathrm{d} \sigma
\end{aligned}
$$

with the following system of the adjoint $p(\mathbf{x}) \in C\left(\Omega^{\mathrm{MC}}\right)$

$$
\begin{aligned}
& -\nabla \cdot(\lambda \nabla p)-c \cdot p=-\frac{\partial j}{\partial T}(\cdot, T) \quad \text { in } \Omega^{\mathrm{MC}} \backslash \Gamma^{\mathrm{int}}, \\
& \lambda^{\mathrm{ex}} \frac{\partial p}{\partial \mathbf{n}}+\left(\alpha^{\prime}(T)\left(T-T^{\mathrm{amb}}\right)+\alpha(T)\right) p=0 \quad \text { on } \Gamma^{\mathrm{ex}}, \\
& {[p]_{ \pm}=0 \text { and }\left[\lambda \frac{\partial p}{\partial \mathbf{n}}\right]_{ \pm}=0 \quad \text { on } \gamma \in \Gamma^{\text {int }}}
\end{aligned}
$$

Proof Following [6], differentiation of $J(\Omega)$ in the direction $\mathbf{V}$ leads, in terms of the local shape derivative $(5)$, to

$$
\begin{aligned}
\delta J(\Omega)[\mathbf{V}] & =\int_{\Omega^{\mathrm{MC}}} \frac{\partial j}{\partial T}(\mathbf{x}, T) \cdot \delta T[\mathbf{V}] \mathrm{d} \mathbf{x} \\
& +\sum_{\gamma \in \Gamma^{\text {int }}} \int_{\gamma}\left\langle\mathbf{V}, \mathbf{n}_{\mathrm{e}}\right\rangle j(\mathbf{x}, T)+\left\langle\mathbf{V}, \mathbf{n}_{\mathrm{i}}\right\rangle j(\mathbf{x}, T) \mathrm{d} \sigma .
\end{aligned}
$$

With the adjoint system (7), we get

$$
\begin{aligned}
\delta J(\Omega)[\mathbf{V}] & =\int_{\Omega^{\mathrm{MC}}}(\nabla \cdot(\lambda \nabla p)+c p) \delta T[\mathbf{V}] \mathrm{d} \mathbf{x} \\
& +\sum_{\gamma \in \Gamma^{\mathrm{int}}} \int_{\gamma}\left\langle\mathbf{V}, \mathbf{n}_{\mathrm{e}}\right\rangle j(\mathbf{x}, T)+\left\langle\mathbf{V}, \mathbf{n}_{\mathrm{i}}\right\rangle j(\mathbf{x}, T) \mathrm{d} \sigma .
\end{aligned}
$$


As $\mathbf{n}_{\mathrm{e}}=-\mathbf{n}_{\mathrm{i}}$, the integrals over the interface $\gamma \in \Gamma^{\mathrm{int}}$ cancel out each other. Application of Green's formula then provides

$$
\begin{aligned}
\delta J(\Omega)[\mathbf{V}] & =\int_{\Omega^{\mathrm{MC}}} \underbrace{(\nabla \cdot(\lambda \nabla \delta T[\mathbf{V}])+c \cdot \delta T[\mathbf{V}]) p}_{=0} \mathrm{~d} \mathbf{x} \\
& +\int_{\Gamma^{\mathrm{ex}}} \underbrace{\left(\lambda^{\mathrm{ex}} \frac{\partial p}{\partial \mathbf{n}} \delta T[\mathbf{V}]-\lambda^{\mathrm{ex}} \frac{\partial \delta T[\mathbf{V}]}{\partial \mathbf{n}} p\right)}_{=0} \mathrm{~d} \sigma \\
& +\sum_{\gamma \in \Gamma^{\mathrm{int}}}\left[\int_{\gamma}\left(\lambda^{\mathrm{i}} \frac{\partial p^{\mathrm{i}}}{\partial \mathbf{n}_{\mathrm{i}}} \delta T^{\mathrm{i}}[\mathbf{V}]-\lambda^{\mathrm{i}} \frac{\partial \delta T^{\mathrm{i}}[\mathbf{V}]}{\partial \mathbf{n}_{\mathrm{i}}} p^{\mathrm{i}}\right) \mathrm{d} \sigma\right. \\
& =\sum_{\gamma \in \Gamma^{\mathrm{int}}}\left[\int_{\gamma} \lambda^{\mathrm{e}} \frac{\partial p^{\mathrm{e}}}{\partial \mathbf{n}}\left[\delta T[\mathbf{V}] \lambda_{ \pm}^{\mathrm{e}} \frac{\partial p^{\mathrm{e}}}{\partial \mathbf{n}_{\mathrm{e}}} \delta T^{\mathrm{e}}[\mathbf{V}]-\lambda^{\mathrm{e}} \frac{\partial \delta T^{\mathrm{e}}[\mathbf{V}]}{\partial \mathbf{n}_{\mathrm{e}}} p^{\mathrm{e}}\right) \mathrm{d} \sigma p_{\gamma}^{\mathrm{e}}\left[\lambda \frac{\partial \delta T[\mathbf{V}]}{\partial \mathbf{n}}\right]_{ \pm} \mathrm{d} \sigma\right] .
\end{aligned}
$$

Replacing the jumps in the Dirichlet data and the Neumann data by the interface conditions in (5) results in

$$
\begin{aligned}
\delta J(\Omega)[\mathbf{V}]= & \sum_{\gamma \in \Gamma^{\mathrm{int}}} \int_{\gamma}-\lambda^{\mathrm{e}} \frac{\partial p^{\mathrm{e}}}{\partial \mathbf{n}}\langle\mathbf{V}, \mathbf{n}\rangle\left[\frac{\partial T}{\partial \mathbf{n}}\right]_{ \pm} \\
& -p^{\mathrm{e}}\left(\operatorname{Div}\left(\langle\mathbf{V}, \mathbf{n}\rangle[\lambda]_{ \pm} \nabla_{\tau} T^{\mathrm{e}}\right)+\left([c]_{ \pm} T^{\mathrm{e}}+[f]_{ \pm}\right)\langle\mathbf{V}, \mathbf{n}\rangle\right) \mathrm{d} \sigma .
\end{aligned}
$$

Integration by parts on the interface boundaries

$$
-\int_{\gamma} p^{\mathrm{e}} \operatorname{Div}\left(\langle\mathbf{V}, \mathbf{n}\rangle[\lambda]_{ \pm} \nabla_{\tau} T^{\mathrm{e}}\right) \mathrm{d} \sigma=\int_{\gamma} \nabla_{\tau} p^{\mathrm{e}} \nabla_{\tau} T^{\mathrm{e}}\langle\mathbf{V}, \mathbf{n}\rangle[\lambda]_{ \pm} \mathrm{d} \sigma
$$

finally implies the desired Hadamard representation of the shape gradient.

\section{Algorithmic and numerical implementation}

First, we present our general optimization strategy that depends on the density and number of single cables in the multicable. Moreover, we introduce procedures to create appropriate initial multicable configurations and describe the implementation of the shape functional as well as the routine to compute the jumps in the Neumann data. Finally, we explain how the entire algorithm, including gradient based shape optimization and genetic algorithm, is implemented. 
5.1 Optimization strategy

To solve the optimal multicable layout problem (3), we employ shape optimization, a genetic algorithm and combine both optimization techniques. The most important part of the entire algorithm, which will be provided in Section 5.5, is the combination of both techniques (illustrated in Fig. 5). Therein, we use $M$ different initial cable configurations, obtained by the application of a so called cable squeezing algorithm [20] (for details, cf. Section 5.2).

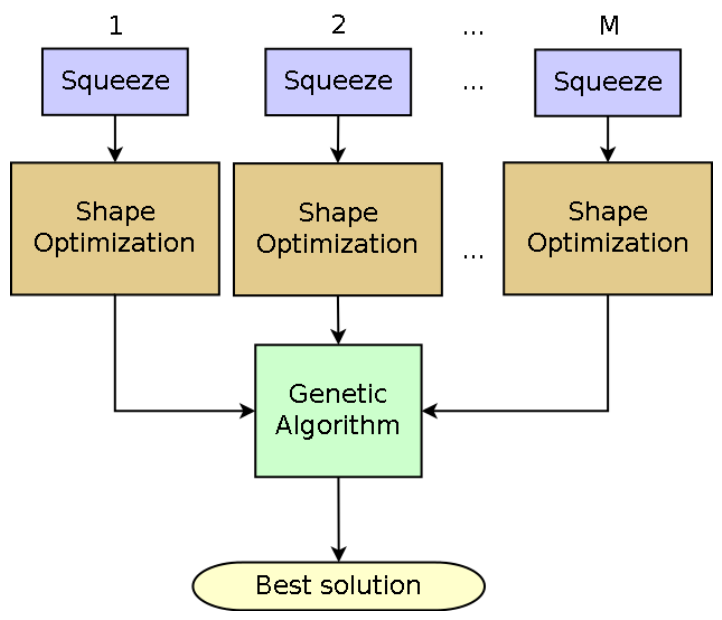

Fig. 5: Overview of the general optimization strategy to solve the optimal multicable layout problem.

Applying shape optimization to these initial configuration yields local minima. However, those do not always represent a satisfying solution of the present problem. For this reason, we pass our $M$ local minima to a genetic algorithm (cf. Sections 3.2 and 5.5.2), which tries to find a better solution.

The coupling of these different algorithms is adequate, as an exclusive use of the genetic algorithm is too time extensive and expensive. Furthermore, we obtain much better results in our simulations with the mixed strategies. On the other hand, for very densely packed cables, the profit of shape optimization is small. Hence, only the cable squeezing and genetic algorithm have been applied to these cases, yet.

\subsection{Initialization}

As mentioned, different local minima may exist for multicables that consist of several single cables. In order to ensure feasibility of the cable configurations, we apply a cable squeezing algorithm (cf. [20, Algorithm 1]). It simulates the 
cable production process of multicables. Therein, each single cable is initially assigned to a template position (cf. [20, Fig. 3]). These are arranged in a circular shape around the centre and numbered ascendantly, starting with number 1 in the centre. Assigning each single cable to a template position (theoretically) yields multicable configurations with extremely large (air) gaps. Thus, the single cables are squeezed afterwards, which simulates how cables are pressed together in productions. After a fixed number of squeezing steps, the diameter of the surrounding circle, that includes all single cable circles, has to be smaller than or equal to the given inner diameter of the exterior insulation. If it is not the case, this initial template configuration cannot be used for the optimization.

The initial template assignment finally has an important influence on the computed local minimum. Thus, we propose different heuristic strategies to create good assignments of the single cables to their template positions. They depend on the current density $J_{k}=I_{k} / A_{k}, k=1, \ldots, n$, of each single cable, with $I_{k}$ the electric current and $A_{k}$ the cross sectional area of the metallic part of single cable $k$ :

- The single cables are assigned to the template positions in descending order with respect to $J_{k}$, denoted by INL;

- The single cables are assigned to the template positions in ascending order with respect to $J_{k}$, denoted by OUL;

- The single cables are ordered ascendantly with respect to $J_{k}$, but assigned to the template positions in steps of $2,3,4$ respectively 5 , denoted by OUL2, OUL3, OUL4 and OUL5;

- The single cables are assigned to template positions such that two cables following each other in order dependent on $J_{k}$ have the greatest possible distance in the template, denoted by OPP;

- Further template configurations are completely arbitrary and denoted by $\mathrm{MC} 1, \mathrm{MC} 2, \ldots$.

By tendency, hotter single cables can give more thermal energy to the ambience if they are positioned nearer to the exterior boundary. ${ }^{2}$ Hence, especially OUL, OUL2-OUL5 and OPP seem promising to give good configurations. As there exist cases for which other template configurations yield our best solution, we added INL and the arbitrary assignments.

\subsection{Shape functional}

The aim of our calculations is to minimize the maximum temperature in a multicable. As the functional

$$
\min _{\Omega \in \mathcal{O}_{\mathrm{ad}}}\|T\|_{L^{\infty}\left(\Omega^{\mathrm{MC}}(\Omega)\right)}=\min _{\Omega \in \mathcal{O}_{\mathrm{ad}}} \sup _{\overline{\mathbf{x}} \in \Omega^{\mathrm{MC}}(\Omega)}|T(\overline{\mathbf{x}})|
$$

\footnotetext{
2 Note that assuming the cables to be surrounded by air is a standard in the norms for dimensioning of cables. Thus, we also suppose the multicable to be hanging in free air in our examples.
} 
is not differentiable and thus the shape optimization approach would not be applicable, we define $J$ by

$$
J(\Omega):=\min _{\Omega \in \mathcal{O}_{\mathrm{ad}}} \frac{1}{q}\|T\|_{L^{q}\left(\Omega^{\mathrm{MC}}(\Omega)\right)}^{q}=\min _{\Omega \in \mathcal{O}_{\mathrm{ad}}} \frac{1}{q} \int_{\Omega^{\mathrm{MC}}(\Omega)}|T(\mathbf{x})|^{q} \mathrm{~d} \mathbf{x}
$$

for fixed values of $q>0$. In (7), the derivative of the objective function appears on the right hand side of the PDE. For the sake of convenience, we introduced the factor $1 / q$ and the exponent $q$. Both do hardly influence the optimization process. As the right hand side could explode for too large values of $q$ which results in numerical problems, we use values of $q=2,3,4$ or 5 in general. ${ }^{3}$

\subsection{Computation of jumps in Neumann data}

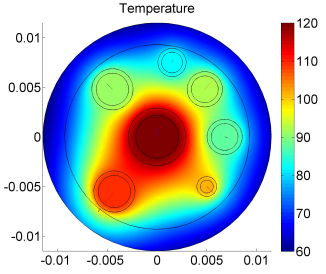

(a)

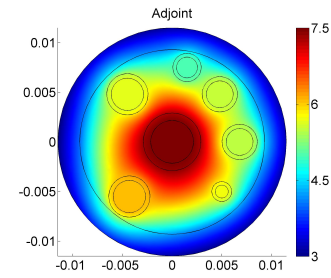

(b)

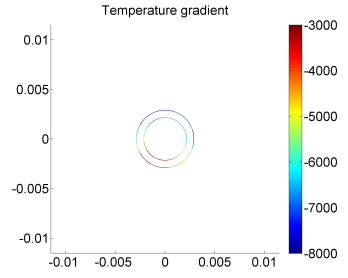

(c)

Fig. 6: (a) Temperature distribution and (b) associated adjoint solution in a multicable, and (c) temperature gradient on the boundaries of a single cable.

Figs. 6, 7 and 8 illustrate the main quantities computed to determine the jumps in the Neumann data (cf. Fig. 6c) with COMSOL Multiphysics 3.5a for an exemplary multicable consisting of seven single cables. Apart from the calculation of the temperature profile (Fig. 6a) and the adjoint (Fig. 6b), we have to project the temperature distribution on the single cable core (Fig. 7a), its complement (Fig. 7b), the single cable insulation (Fig. 8a) and also its complement onto separate domains (Fig. 8b). This is required because we obtain completely wrong results if we directly compute the temperature distribution and the difference of the derivatives to the outer and inner normal in COMSOL. It is due to an internal smoothing of the function in the software. To deal with the problem, the derivative to the outer normal is computed on the temperature projected onto the domains (core respectively insulation of

\footnotetext{
3 Alternatively, the factor $1 / q$ and the exponent $q$ could be dropped in the shape functional. Then, an approximation of the $L^{\infty}$-norm would be computed for large $q$, with the drawback of a more complicated right hand side of the adjoint system.
} 
the single cable) and to the inner normal on their complements. This procedure has to be performed for every single cable and finally yields correct gradients, which was doublechecked with finite differences.

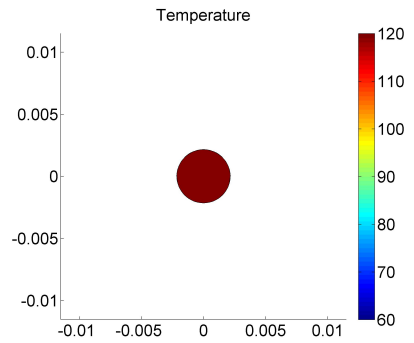

(a)

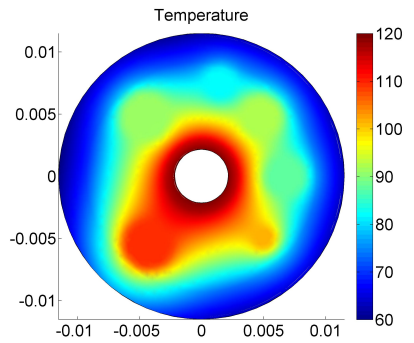

(b)

Fig. 7: Temperature distribution on (a) a single cable core and (b) on its complement.

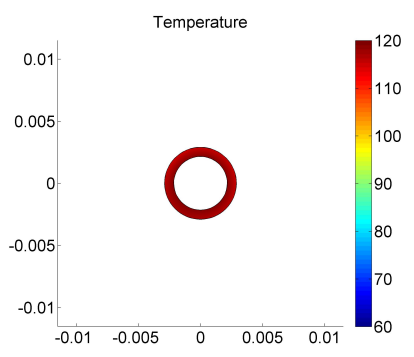

(a)

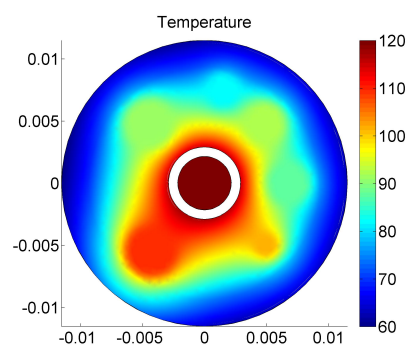

(b)

Fig. 8: Temperature distribution on (a) a single cable insulation and (b) on its complement.

Note that the local translation of single cable $k$ in direction of the $x$ coordinate is described by

$$
\mathbf{V}(\mathbf{x})=\mathbb{I}_{\Omega_{k}}(\mathbf{x})\left(\begin{array}{ll}
1 & 0 \\
0 & 0
\end{array}\right) \mathbf{x}
$$

and the corresponding translation in direction of the $y$-coordinate by

$$
\mathbf{V}(\mathbf{x})=\mathbb{I}_{\Omega_{k}}(\mathbf{x})\left(\begin{array}{ll}
0 & 0 \\
0 & 1
\end{array}\right) \mathbf{x}
$$


with $\mathbb{I}(\cdot)$ denoting the indicator function.

Table 1 essentially shows the accordance resp. discrepancy of the adjoint method and finite differences in the computation of the shape gradient for the present example with seven single cables. The parameters $h$ and hnumedg ${ }^{4}$ represent measures for the mesh refinement on the entire domain of the multicable and for the refinement on the edges. Smaller values for $h$ and larger values for hnumedg result in finer grids and larger numbers of degrees of freedom. The maximum of absolute discrepancies $\max \left(\mathrm{err}_{\mathrm{abs}}\right)$ between adjoint and finite difference method is obtained in the partial derivative to the component Comp. The maximum relative discrepancy $\max \left(\mathrm{err}_{\mathrm{rel}}\right)$ does not have to occur in the same component. It represents the maximum of deviations divided by the value of the derivative obtained via finite differences in all components. The quantities $t_{\mathrm{ADJ}}$ and $t_{\mathrm{FD}}$ specify the time in seconds necessary for the gradient determination with each method, $\Delta t$ their difference in time.

\begin{tabular}{ccccccc}
\hline$h$ (hnumedg) & DOF & $\max \left(\mathrm{err}_{\mathrm{abs}}\right)[\mathrm{Comp}]$ & $\max \left(\mathrm{err}_{\mathrm{rel}}\right)$ & $t_{\mathrm{ADJ}}[s]$ & $t_{\mathrm{FD}}[s]$ & $\Delta t[s]$ \\
\hline $5(10)$ & 26002 & $6.712\left[x_{5}\right]$ & $1.06 \mathrm{e}-2$ & 25.8 & 560.4 & 534.6 \\
$5(20)$ & 70842 & $2.050\left[y_{4}\right]$ & $3.75 \mathrm{e}-3$ & 44.7 & 1059.1 & 1024.2 \\
$4(30)$ & 148730 & $1.332\left[y_{4}\right]$ & $1.25 \mathrm{e}-3$ & 98.9 & 2704.2 & 2605.3 \\
\hline
\end{tabular}

Table 1: Statistics for the mesh refinements, degrees of freedom, absolute resp. relative discrepancies between adjoint and non-adjoint method as well as calculation times for a specific example with seven single cables.

We observe a good accordance of the gradients, calculated via the adjoint method and finite differences with $\epsilon=1.0 \mathrm{e}-4$, especially for larger numbers of degrees of freedom. Thus, to have a reliable gradient approximation, we have to use fine meshes. Furthermore, the computation times by the adjoint method are much shorter than those of the finite difference method. We have to admit that the latter, neither the method itself, nor the implementation, is optimized. Nevertheless, it is obvious that the adjoint method works much faster in this scope because, in addition to the one solution of the state system, the adjoint system, which is only linear ${ }^{5}$, has to be solved once, independent of the number of single cables. In contrast, the nonlinear state system must be solved twice for each optimization variable with central differences (once if we used forward or backward differences), which corresponds to solving the PDE 28 times in this example.

\subsection{Computational algorithm}

The entire optimization algorithm (cf. Fig 9) was implemented in Matlab, version 7.10.0.499. First, geometrical and physical parameters are read from input

\footnotetext{
4 The measure of refinement is a convention used in COMSOL Multiphysics 3.5a.

5 Even the exterior boundary condition is only linear in $p$.
} 
files and convergence criteria of the algorithm, if necessary of the shape optimization procedure and the genetic algorithm, are defined. Depending on the filling factor $F$ of the multicable and the number $N$ of single cables, different strategies are proposed.

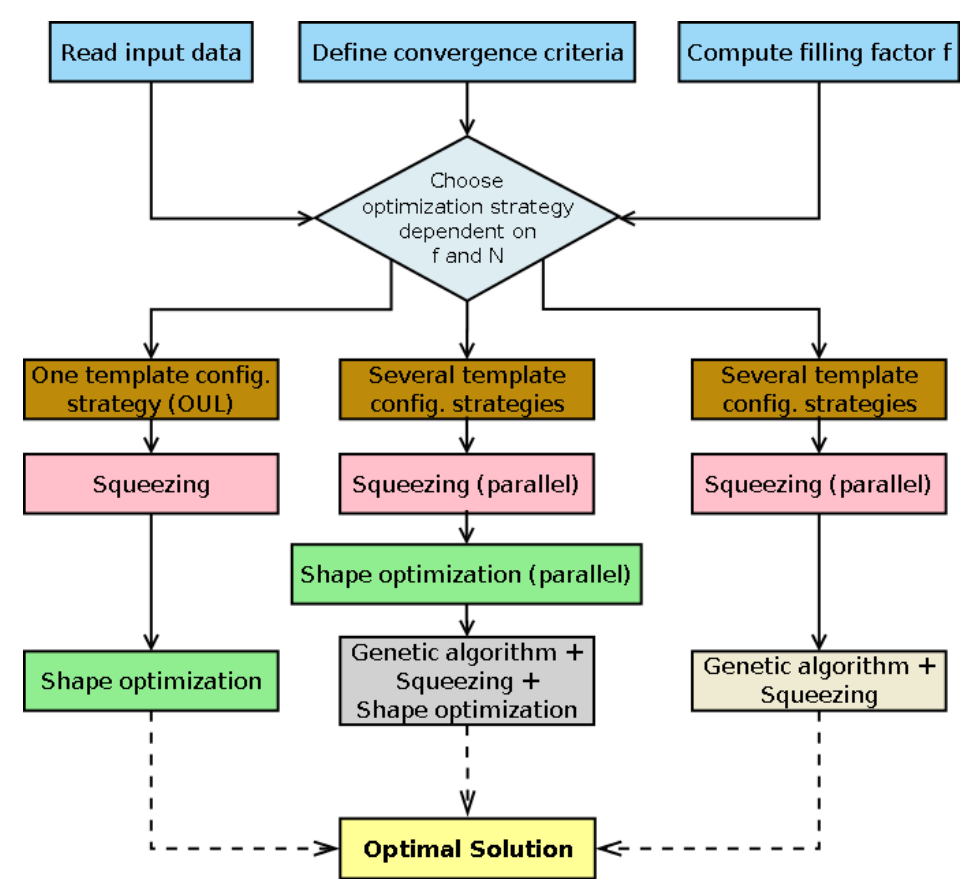

Fig. 9: Program flowchart of the entire multicable optimization procedure.

In case of a small number of single cables and enough space, we use only one initial template configuration. By experience, we recommend OUL. After the squeezing algorithm (implemented in Matlab), one shape optimization procedure in IPOPT is performed, yielding the (approximately) best solution.

For cables that are packed very densely (filling factors larger than 90\%), shape optimization does not make sense. Instead, we use the presented initial template configurations to produce initial multicable configurations and apply the squeezing algorithm. The generated configurations are given to the genetic algorithm, where new configurations are produced and made feasible by squeezing (for details, cf. Section 5.5.2). This procedure, also implemented in Matlab, takes extremely long and in some cases, it does not provide a better solution than the best inital configuration. Improving this part of the algorithm will be subject of future work.

Moderately packed multicables with several single cables, which are common in practice, require a quite complex procedure. It was described in Section 5.1. Note that creating the initial configurations (especially the squeezing 
part), but also several ones during the genetic optimization, are evaluated in parallel in order to save time.

\subsubsection{Implementation of the Shape Optimization Algorithm}

The shape optimization procedure for a given configuration of single cables requires solutions of the state system (1) and the adjoint system (7). Both are computed with the help of COMSOL Multiphysics 3.5a, controled via a Matlab script. Therein, an automatic mesh generator is applied, as well as a damped Newton method [7] to solve the nonlinear PDE system. In every Newton step, we use UMFPACK $[8,9]$ or PARDISO $[24,25]$ for the solution of the linearized systems. For further details, we refer to $[5,20]$.

Afterwards, the shape gradient (6) is evaluated for variations of each single cable in directions of the $x$ - and $y$-axis (except for single cable 1 that only varies in the $y$-coordinate). The corresponding mappings $\mathbf{V}$ for variation of single cable $k$ in the $x$ - resp. $y$-coordinate are indicated in Section 5.4.

Shape gradient, solution of the state resp. adjoint system, the geometrical constraints of the optimization problem, the constraints' derivatives and the initial configuration of the multicable are passed to IPOPT, which then computes a local minimum. ${ }^{6}$ More precisely, we give a vector of size $2 n_{k}$ to IPOPT that contains the sensitivities of the shape functional with respect to the domain variations. In the first component, the sensitivity of $J$ for a local translation of single cable 1 in direction of the $x$-coordinate is given, in the second component the sensitivity of $J$ for a local translation of single cable 1 in direction of the $y$-coordinate, in the third component the sensitivity of $J$ for a local translation of single cable 2 in direction of the $x$-coordinate, etc. Thus, IPOPT - which is indeed an optimizer for finite dimensional problems - has to solve a finite dimensional optimization problem with the midpoints of the single cables as optimization variables.

\subsubsection{Implementation of the Genetic Algorithm}

We briefly state the main aspects of the genetic algorithm's implementation:

\section{- Initialization:}

As explained in Section 5.2, several initial configurations are generated by squeezing of the single cables. If for one of the presented assignment strategies the squeezing does not yield a feasible configuration after a fixed number of squeezing steps, the assignment strategy is skipped and replaced by a further stochastic one. If after trying a certain number of initial strategies, no feasible configuration is attained (e.g. if the exterior diameter is too

6 IPOPT is a software package for large-scale nonlinear optimization that implements an interior-point line-search filter method (cf. [28-30]). In our options, we use a monotone strategy for the barrier parameter $\mu$ of the logarithmic barrier function. If this barrier parameter is small enough and the KKT conditions are fulfilled satisfactorily, an optimum is reached. Otherwise, if no minimum is attained after a given number $n_{\max }$ of iterations, the procedure is interrupted. 
small for the single cables), the algorithm is interrupted and the problem cannot be solved. However, if the number of computed feasible configurations is less than the intended number of individuals of each generation, we continue with fewer individuals.

\section{- Selection:}

The best individual - the configuration with the smallest value of the objective function - is directly passed to the next generation as the elite. $70 \%$ of the population of the next generation are created by crossover, the rest by mutation.

\section{- Genetic operators:}

For crossover, we take the single cable coordinates of two randomly selected parents (multicables) of the previous generation. To generate one child of the next generation, we select a part of the genes (coordinates) from parent 1 , the others from parent 2 . To generate a certain coordinate of a child by mutation, we select a random number of individuals and compute the mean of this coordinate of the chosen individuals, supplemented by further, randomly generated coordinates. This procedure is performed for each coordinate of the child.

Both, crossover and mutation, normally provide multicable configurations that are not feasible. Either, single cables are situated outside of the multicable domain (which is rather rare) or single cables overlap (very frequent). The cable squeezing algorithm of [20] does not only squeeze the single cables, but also removes overlappings of single cables. Thus, after generation of coordinates by crossover and mutation, we apply this algorithm to create feasible, (hopefully) better configurations.

\section{- Termination:}

Normally, many generations are produced within a genetic algorithm. In our approach, a good solution is already approximated after a few generations in most cases. This is due to the combination with shape optimization. For that reason, our genetic algorithm finishes after a (small) number of generations $(\leq 10)$.

\section{Numerical results}

To test our proposed algorithm, we optimize the shape of different multicables, consisting of 1, 3, 15 and 33 single cables. In case of 1 and 3 single cables, the first strategy with exclusive application of shape optimization is sufficient. For 15 and 33 single cables, we use the second strategy. An example for the third strategy is not presented, because it employs no gradient based shape optimization and it is only applied to very specific multicables. All the following examples were carried out in Matlab, version 7.10.0.499, on a Pentium III Xeon processor with 4 cores (each $2.50 \mathrm{GHz}$ ) and in total $32 \mathrm{~GB}$ RAM. 


\subsection{First example}

We first optimize the position of only one single cable in the multicable that has a low heat conductivity in the gaps and an exterior insulation of PVC. The single cable carries a current of $I_{1}=102 \mathrm{~A}$ and its cross sectional area is $A_{1} \approx 14.5 \mathrm{~mm}^{2}$. We suppose the ambient temperature to be $33.2^{\circ} \mathrm{C}$. In the shape functional, we set $q=2$.

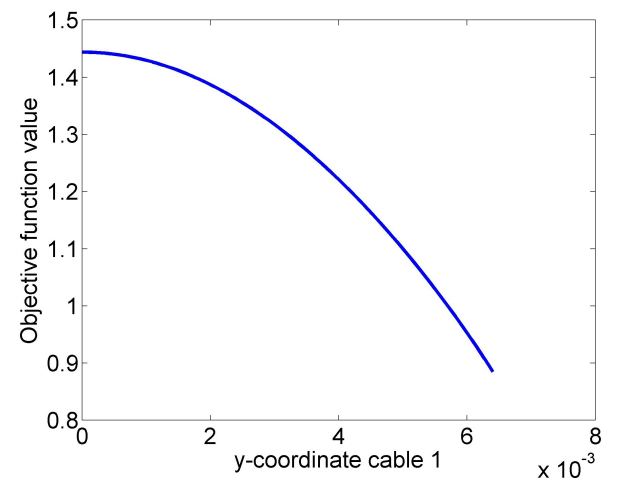

Fig. 10: Objective function values for variation of the $y$-coordinate of one single cable.

As the $x$-coordinate of the midpoint of the first single cable is fixed to zero and the $y$-coordinate $>0$, the objective function value is monotonically decreasing for larger values of the $y$-position of the first single cable (cf. Fig. $10)$. The highest value is obtained at the origin of the coordinate system $(J(\Omega) \approx 1.4439)$, the lowest at the exterior boundary $(J(\Omega) \approx 0.8866)$. Table 2 shows the optimization progress in IPOPT with $\Delta \mathbf{x}$ denoting the Euclidean norm of the step size of the optimization variable in each iteration. F-count indicates the number of function evaluations in each iteration. The computation time is about $328 \mathrm{~s}$ with $\approx 45000$ degrees of freedom in the linear system of every Newton iteration.

Obviously, the optimization works very well. During the optimization process, the single cable, starting from the origin of the coordinate system (Fig. 11a), moves towards the exterior boundary (Fig. 11c). Fig. 11 depicts the temperature distribution for three different configurations, each generated during the optimization process.

Although with $q=2$, our objective function $J(\Omega)$ does not approximate the $L^{\infty}(\Omega)$-norm very accurately, the maximum temperature is much lower in the optimized multicable $\left(\approx 111.8^{\circ} \mathrm{C}\right)$ than in the initial configuration $(\approx$ $\left.147.9^{\circ} \mathrm{C}\right)$. Indeed, if the single cable is situated nearer to the exterior boundary, more heat is emitted to the environment by convection and radiation than it is the case if the hotspot is situated in the centre of the multicable. 


\begin{tabular}{cccc}
\hline Iter & F-count & $J(\Omega)$ & $\Delta \mathbf{x}$ \\
\hline 0 & 1 & 1.4438692 & \\
1 & 1 & 0.8963279 & $3.93 \mathrm{e}-2$ \\
2 & 1 & 1.2103676 & $8.38 \mathrm{e}-3$ \\
3 & 1 & 1.0596074 & $1.20 \mathrm{e}-3$ \\
4 & 1 & 0.9627317 & $7.63 \mathrm{e}-4$ \\
$\vdots$ & $\vdots$ & $\vdots$ & $\vdots$ \\
9 & 4 & 0.8868828 & $1.17 \mathrm{e}-5$ \\
10 & 4 & 0.8868126 & $1.03 \mathrm{e}-5$ \\
11 & 1 & 0.8865531 & $1.45 \mathrm{e}-7$ \\
\hline
\end{tabular}

Table 2: Optimization progress for a multicable with one single cable.

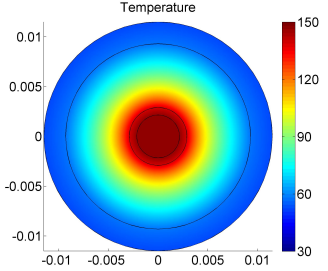

(a)

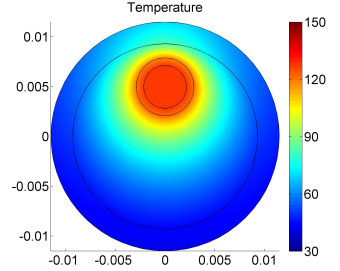

(b)

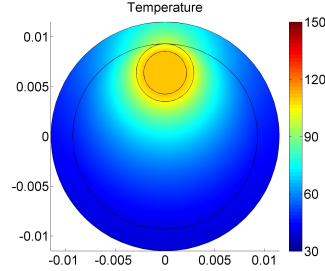

(c)

Fig. 11: Cable configurations generated during the optimization process: (a) Inital configuration with $J(\Omega)=1.44387$, (b) configuration in iteration 3 with $J(\Omega)=1.0596074$, and (c) final configuration with $J(\Omega)=0.88655$.

\subsection{Second example}

Our algorithm is applied to multicables with three single cables. In the first multicable, the current densities are equal for all three single cables, in the second, they vary.

\subsubsection{Multicable with three single cables of equal current loads}

We investigate a multicable consisting of three single cables with equal currents $I_{1}=I_{2}=I_{3}=89 \mathrm{~A}$ and cross sectional areas $A_{1}=A_{2}=A_{3}=14.5 \mathrm{~mm}^{2}$. We set $q=3$.

Starting with an almost regular positioning such that the midpoints of the single cables nearly form an equilateral triangle (cf. Fig. 12a), the single cables move towards the exterior boundary (cf. Figs. 12b-12d). Having reached the boundary (cf. Fig. 12e), they are forced to find a configuration for which the cables have the largest possible distance from each other, i. e. an equilateral triangle of the midpoints with each single cable situated at the boundary of the multicable (cf. Fig. 12f). 


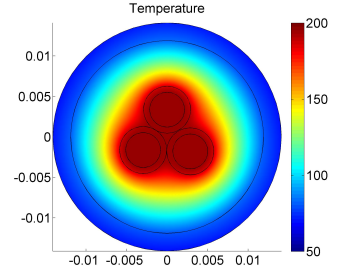

(a)

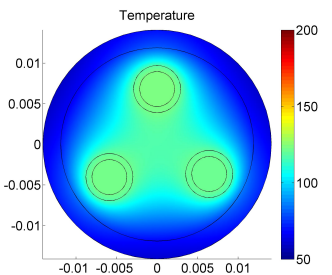

(d)

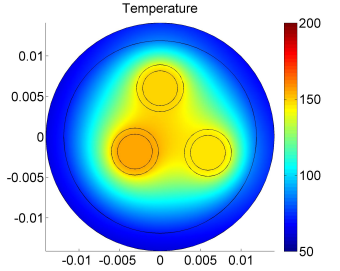

(b)

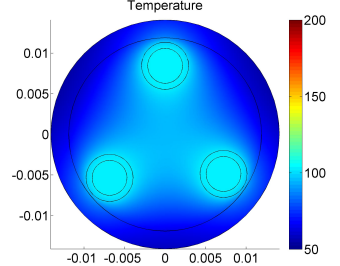

(e)

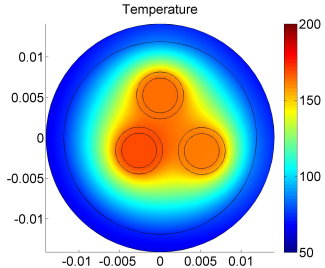

(c)

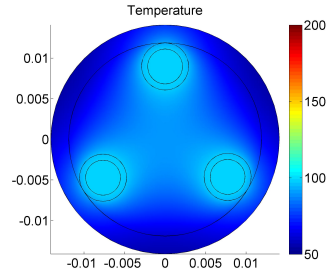

(f)

Fig. 12: Cable configurations generated during the optimization process for a multicable which consists of three single cables carrying equal current loads: (a) Initial configuration, (b) configuration in iteration 1, (c) configuration in iteration 2, (d) configuration in iteration 5, (e) configuration in iteration 9, and (f) configuration in iteration 30 .

In IPOPT, it sometimes happens that the objective function value increases in an iteration, e.g. in our case at the second iteration (cf. Table 3 and Fig. 13). This is due to the interior-point algorithm, when the barrier parameter is modified [28]. The entire optimization is interrupted after thirty iterations, i. e. in this example for $n_{\max }=30$.

Obviously, the maximum temperature decreases from $\approx 197^{\circ} \mathrm{C}$ to $\approx 100^{\circ} \mathrm{C}$ during the optimization process (cf. Table 4). The reason for this diminution is again that more thermal energy can be emitted at the transition to air if the single cables are placed at the exterior boundary. In the final configuration, they have the greatest possible distances from each other.

The insulation material of the single cables and the exterior insulation is supposed to consist of PVC. Assumed a melting point of about $130^{\circ} \mathrm{C}$, the initial multicable would not be able to endure the given current loads, whereas the optimized one could without any problems.

Note that the heat conductivity of the inner insulation is computed according to formula (9) in [20]. Thus, it is supposed to be a mixture of air and solid material. The modelling of the inner heat conductivity with such low filling factors $(F \leq 30 \%)$ might be inadequate for real multicables. If we assume the inner material to consist entirely of solid material, e.g. PVC 


\begin{tabular}{cccc}
\hline Iter & F-count & $J(\Omega)$ & $\Delta \mathrm{x}$ \\
\hline 0 & 1 & $5.0743619 \mathrm{e}+02$ & \\
1 & 2 & $3.0311155 \mathrm{e}+02$ & $5.32 \mathrm{e}-01$ \\
2 & 1 & $3.6448495 \mathrm{e}+02$ & $9.96 \mathrm{e}-03$ \\
3 & 1 & $2.0319573 \mathrm{e}+02$ & $3.25 \mathrm{e}-03$ \\
4 & 1 & $2.1952290 \mathrm{e}+02$ & $3.87 \mathrm{e}-04$ \\
5 & 1 & $1.9638202 \mathrm{e}+02$ & $4.28 \mathrm{e}-04$ \\
$\vdots$ & $\vdots$ & $\vdots$ & $\vdots$ \\
8 & 1 & $1.3197190 \mathrm{e}+02$ & $5.74 \mathrm{e}-05$ \\
9 & 1 & $1.3202247 \mathrm{e}+02$ & $4.14 \mathrm{e}-05$ \\
10 & 1 & $1.2003736 \mathrm{e}+02$ & $4.30 \mathrm{e}-04$ \\
$\vdots$ & $\vdots$ & $\vdots$ & $\vdots$ \\
28 & 11 & $1.1789933 \mathrm{e}+02$ & $2.84 \mathrm{e}-04$ \\
29 & 13 & $1.1789923 \mathrm{e}+02$ & $2.97 \mathrm{e}-04$ \\
30 & 14 & $1.1789918 \mathrm{e}+02$ & $2.98 \mathrm{e}-04$
\end{tabular}

Table 3: Optimization progress for a multicable with three equally loaded single cables.

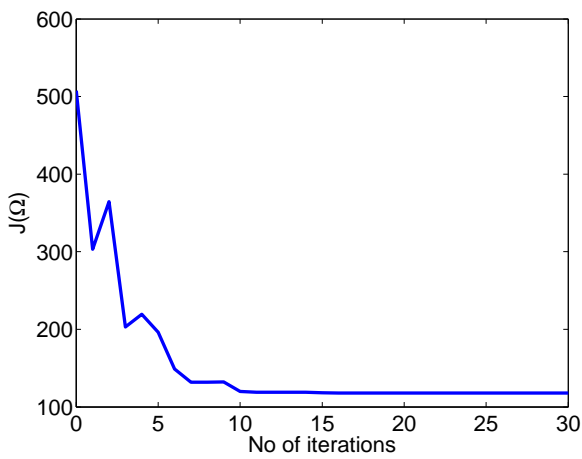

Fig. 13: Convergence history of the optimizer for a multicable with three equally loaded single cables.

$\left(\lambda^{\text {gaps }}=0.19 \mathrm{~W} /(\mathrm{m} \cdot \mathrm{K})\right)$, the maximum temperature decreases by shape optimization from $102.1^{\circ} \mathrm{C}$ to $92.0^{\circ} \mathrm{C}$.

Concerning the calculation expense, most of the time is spent to solve state and adjoint systems during the 63 function evaluations (cf. Table 4 with further statistics). The rather long evaluation times for the systems with about 120000 degrees of freedom (DOFs) and $\approx 30000$ elements (NOE) are explained by the nonlinearities. The squeezing time is nearly negligible and calculation time in the optimizer is very small. In fact, the optimization problem to be solved in the nonlinear optimizer is very small. Neglecting the $x$-coordinate of the first single cable as well as the lower and upper bounds for the other variables, it consists of 5 optimization variables and 6 geometrical constraints. 


\begin{tabular}{llll}
\hline & \multicolumn{3}{c}{ Statistics } \\
\hline Init $\max _{\mathbf{x} \in \Omega} T(\mathbf{x})$ & $196.5^{\circ} \mathrm{C}$ & Final $\max T(\mathbf{x})$ & $99.7^{\circ} \mathrm{C}$ \\
No of iterations & 30 & No of function evaluations & 63 \\
Time optimizer & $\approx 42 \mathrm{~s}$ & Time FEM solver & $\approx 3584 \mathrm{~s}$ \\
Time squeezing & $\approx 1.1 \mathrm{~s}$ & Total time & $\approx 3632 \mathrm{~s}$ \\
DOFs & $\approx 120000$ & NOE & $\approx 30000$ \\
\hline
\end{tabular}

Table 4: Statistics for the optimization of a multicable with three equally loaded single cables.

For higher values of $q$, it is even more expensive to solve the PDEs. With $q=5$, we obtained nearly the same optimization process (with of course different values for $J(\Omega)$ ) and similar calculation times in the optimizer, but the solution of the PDEs took about four times longer for equal mesh refinements.

\subsubsection{Multicable with three single cables of different current loads}

We suppose again $N=3, q=3$, equal cross sectional areas for the single cables and the same material and ambient parameters as in the previous example. The only difference is that, now, the cables carry different current loads of $I_{1}=103 \mathrm{~A}$ and $I_{2}=I_{3}=80 \mathrm{~A}$.

Starting from the same initial configuration as in Section 6.2.1, the cables move towards the exterior border (cf. Fig. 14). In contrast to the previous example, they move until finally the centre coordinates form an isosceles triangle, which is not equilateral (cf. Fig. 14f). The distance to the single cable carrying a higher current is larger than between the cables with equal currents. The objective function value for $q=3$ improves from $J\left(\Omega_{\text {init }}\right) \approx 486.3$ to $J\left(\Omega_{\text {opt }}\right) \approx$ 118.3 , and the maximum temperature decreases from $\max _{\mathbf{x} \in \Omega_{\text {init }}} T(\mathbf{x}) \approx 199.2^{\circ} \mathrm{C}$ to $\max _{\mathbf{x} \in \Omega_{\mathrm{opt}}} T(\mathbf{x}) \approx 115.3^{\circ} \mathrm{C}$.

The entire optimization took 4991 seconds with 30 optimization steps and 84 function evaluations. 42 seconds where required in the nonlinear optimizer, one second for squeezing, the rest to solve the PDEs. The number of elements was about 30000 , corresponding to $\approx 120000$ degrees of freedom in the linearized systems, which were solved by UMFPACK.

\subsection{Third example}

We consider a multicable consisting of 15 single cables with different cross sectional areas $\left(A_{k}\right)$, currents $\left(I_{k}\right)$ and current densities $\left(J_{k}\right)$ listed in Table 5 . The filling factor of the multicable is $F \approx 47 \%$, the ambient temperature $T^{\mathrm{amb}}=33.2^{\circ} \mathrm{C}$ and we use $q=3$. The maximum temperatures of the depicted initial configurations, obtained by squeezing of the initial template configurations (cf. Fig. 15), vary between $142.2-149.7^{\circ} \mathrm{C}$. 


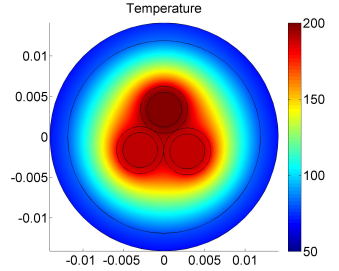

(a)

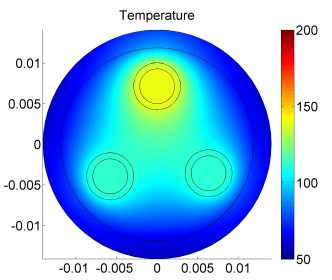

(d)

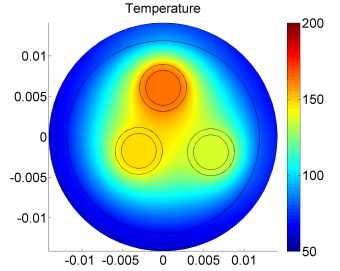

(b)

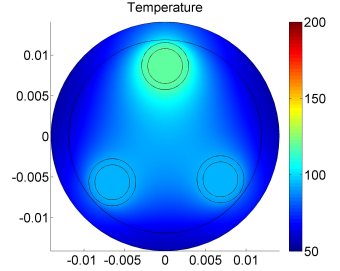

(e)

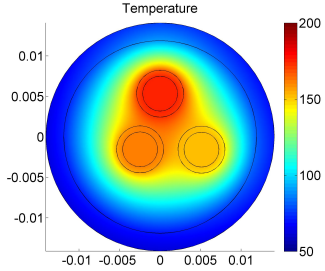

(c)

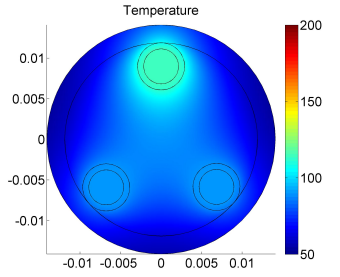

(f)

Fig. 14: Cable configurations generated during the optimization process for a multicable consisting of three single cables carrying different current loads: (a) Initial configuration, (b) configuration in iteration 1, (c) configuration in iteration 2, (d) configuration in iteration 5, (e) configuration in iteration 10 , and (f) configuration in iteration 30 .

The lowest maximum temperature after shape optimization applied to the initial template configurations, thus the maximum of the best individual for the initial generation, is $127^{\circ} \mathrm{C}$. After a total calculation time of nearly $30 \mathrm{~h}$ with about 7100 function evaluations ${ }^{7}$, partly in parallel, we obtain our approximated (global) minimal maximum temperature (cf. Fig. 16a).

Therein, each shape optimization in IPOPT is interrupted after no later than 30 iterations. In each function evaluation, $30000-40000$ elements with $120000-160000$ degrees of freedom are used for the finite element approximation. The linear solver is UMFPACK. The entire optimization problem has 29 optimization variables ( $x$-coordinate of first single cable neglected) with 120 inequality constraints and 435 non-zero entries in the inequality constraint Jacobian.

The minimal maximum temperature of our best cable configuration is $125.9^{\circ} \mathrm{C}$. Hence, in this case with a rather low filling factor, most of the optimization is done by the gradient based shape optimization. The influence of the genetic algorithm is rather small in relation to the computational effort (cf. Fig. 17a and Table 6). But this also shows that the different template configuration strategies, combined with squeezing and shape optimization, al-

\footnotetext{
7 Each function evaluation includes the solution of state and adjoint system.
} 


\begin{tabular}{cccc}
\hline Single Cable No. & $I_{k}(\mathrm{~A})$ & $A_{k}\left(\mathrm{~mm}^{2}\right)$ & $J_{k}\left(A / \mathrm{mm}^{2}\right)$ \\
\hline 1 & 53.00 & 14.5 & 3.65 \\
2 & 82.00 & 8.30 & 9.89 \\
3 & 12.00 & 8.30 & 1.45 \\
4 & 12.00 & 3.46 & 3.46 \\
5 & 52.00 & 8.30 & 6.27 \\
6 & 43.00 & 5.73 & 7.51 \\
7 & 14.88 & 5.73 & 2.60 \\
8 & 16.20 & 5.73 & 2.83 \\
9 & 18.00 & 3.46 & 5.20 \\
10 & 18.00 & 3.46 & 5.20 \\
11 & 9.50 & 1.33 & 7.16 \\
12 & 9.50 & 1.33 & 7.16 \\
13 & 10.00 & 0.68 & 14.72 \\
14 & 4.50 & 0.68 & 6.62 \\
15 & 0.34 & 0.50 & 0.68 \\
& & &
\end{tabular}

Table 5: Electric currents, cross sectional areas of the metallic core and current densities in the 15 single cables of the multicable.

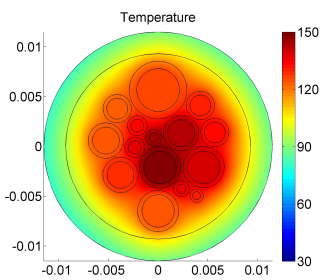

(a) INL $(J(\Omega)=219.4)$.

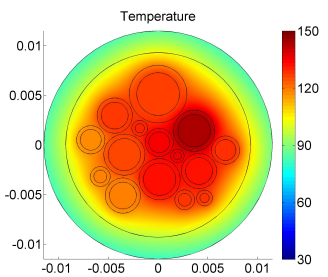

(d) OUL2 $(J(\Omega)=207.2)$.

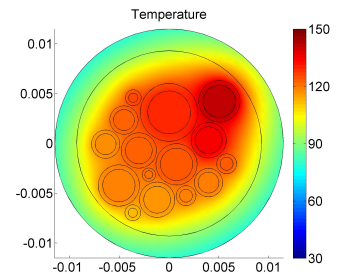

(b) OUL $(J(\Omega)=195.5)$.

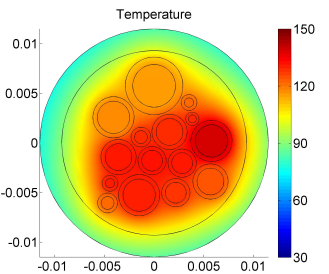

(e) OUL4 $(J(\Omega)=198.0)$.

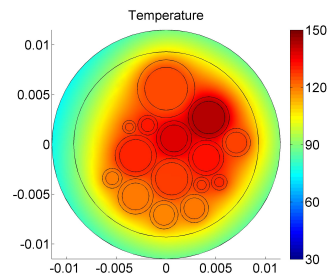

(c) $\operatorname{MCL1}(J(\Omega)=204.1)$.

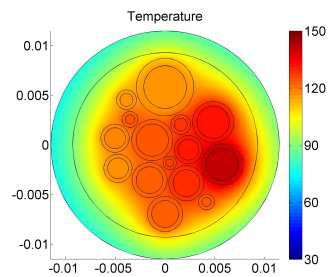

(f) $\operatorname{OPP}(J(\Omega)=193.8)$

Fig. 15: Temperature distributions in multicables consisting of 15 single cables for different initial template assignments.

ready provide a good cable configuration with a maximum temperature only slightly higher than that of the optimal multicable. 


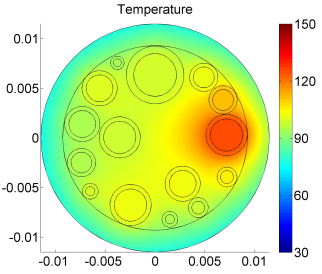

(a)

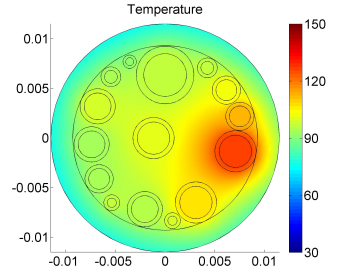

(b)

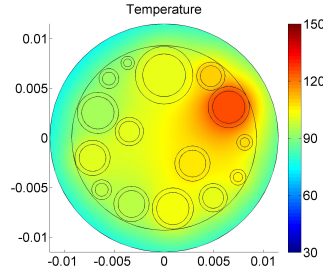

(c)

Fig. 16: Improved cable configurations obtained by application of the genetic algorithm for 15 single cables: (a) Best GA configuration with $J(\Omega)=134.9$, (b) second GA configuration with $J(\Omega)=135.0$, and (c) third GA configuration with $J(\Omega)=137.0$.

\begin{tabular}{cccccccccc}
\hline $\begin{array}{c}\text { Generation } \\
\text { No. }\end{array}$ & 1 & 2 & 3 & 4 & 5 & 6 & 7 & 8 & 9 \\
\hline 0 & 154.0 & 136.7 & 137.7 & 139.8 & 147.0 & 141.0 & 157.5 & 136.5 & 140.7 \\
1 & 136.5 & 143.6 & 140.3 & 141.5 & 147.9 & 142.8 & 142.4 & 136.2 & 143.1 \\
2 & 136.2 & 141.7 & 142.3 & 147.8 & 140.3 & 142.0 & 137.0 & 142.3 & 142.8 \\
3 & 136.2 & 135.8 & 137.6 & 142.6 & 135.8 & 150.8 & 147.8 & 144.2 & 149.6 \\
4 & 135.8 & 134.9 & 138.8 & 140.6 & 138.8 & 140.1 & 146.4 & 146.1 & 137.3 \\
5 & 134.9 & 137.0 & 141.8 & 135.0 & 146.6 & 141.7 & 140.4 & 143.4 & 140.2 \\
\hline
\end{tabular}

Table 6: Fitness values of all individuals for 6 generations in the progress of the genetic algorithm for multicables with 15 single cables.

\subsection{Fourth example}

Finally, we optimize the multicable that presented the motivation for our paper. It consists of 33 single cables. We drop the explicit specification of all current loads and cross sectional areas. Instead, Fig. 18 depicts the current density of each single cable. Furthermore, we use $T^{\mathrm{amb}}=33.2{ }^{\circ} \mathrm{C}$ and $q=3$. The filling factor of the multicable is $F \approx 63 \%$ with an inner diameter of $19.2 \mathrm{~mm}$ and outer diameter of $23.6 \mathrm{~mm}$ for the exterior insulation.

Fig. 19 shows that the maximum temperature obtained by the initial template configuration INL (Fig. 19a) is $108.5^{\circ} \mathrm{C}$. In contrast, our optimized cable has a maximum temperature of $92.4^{\circ} \mathrm{C}$ (Fig. 20a). That means that the difference in maximum temperatures between a bad and good configuration for this case can be about $16.1 \mathrm{~K}$. Thus, less ambient temperature, the reduction of maximum temperature of the optimized cable compared to that obtained with INL is $\approx 21 \%$.

In the optimization procedure of the genetic algorithm, we determined 6 generations, each consisting of 9 individuals (cf. Table 7 ). Therein, the objective function value was reduced from $J\left(\Omega_{\text {init }}\right)=93.76$ to $J\left(\Omega_{\mathrm{opt}}\right)=92.43$ (cf. Fig. 17b). The shape optimization of each individual in IPOPT was interrupted 


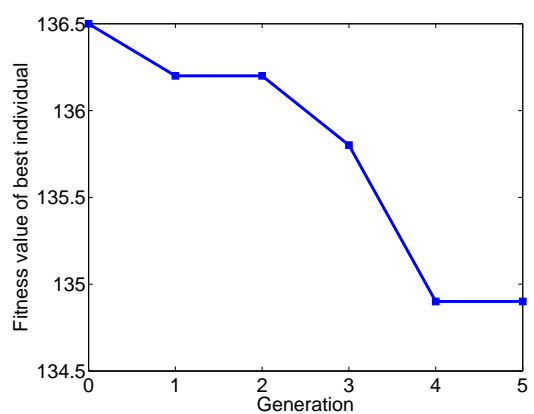

(a)

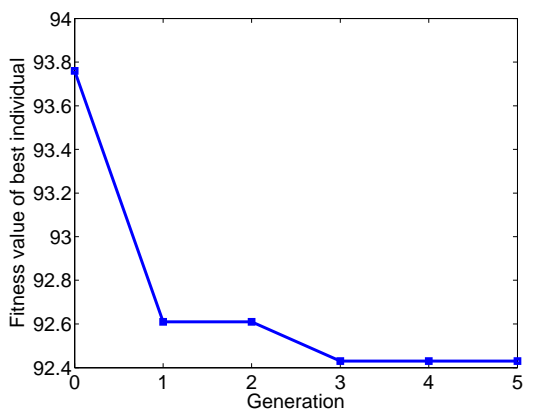

(b)

Fig. 17: Convergence history of the genetic algorithm for multicables with (a) 15 single cables and (b) 33 single cables.

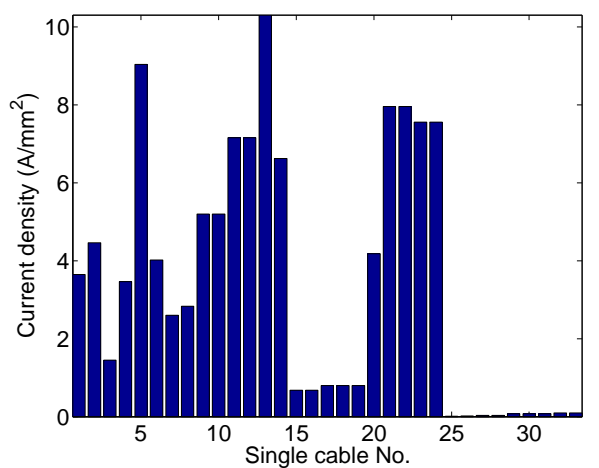

Fig. 18: Current densities of the 33 single cables in the multicable.

after latest $n_{\max }=50$. The number of optimization variables was 65 with 561 inequality constraints and 2145 non-zero entries in the inequality constraint Jacobian. The linearised systems in the evaluation with finite elements were solved with PARDISO and had between 250000-350000 unknowns.

Altogether, the state and adjoint systems were evaluated about 7300 times. The entire optimization process took about 42 hours.

\section{Conclusion}

The goal of this paper was to derive an algorithm for the optimization of current carrying multicables. This was enabled by coupling a cable squeezing algorithm, helping to generate feasible cable configurations, to a genetic algorithm and a gradient based shape optimization approach. The positive influence of the gradient based shape optimization for multicables consisting 


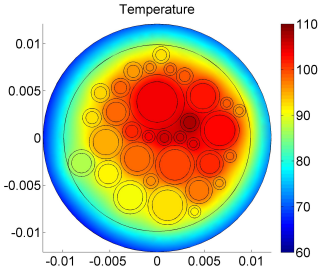

(a) INL $(J(\Omega)=116.2)$.

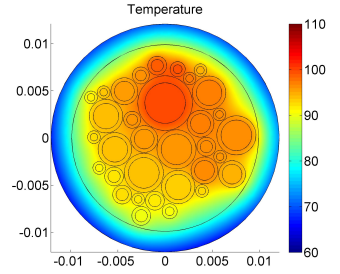

(b) OUL $(J(\Omega)=108.3)$.

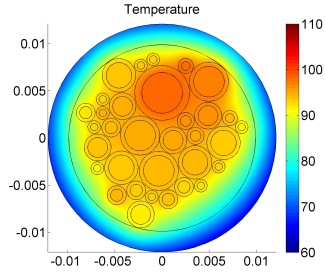

(c) $\operatorname{OPP}(J(\Omega)=106.1)$.

Fig. 19: Temperature distributions in multicables consisting of 33 single cables for different initial template assignments.

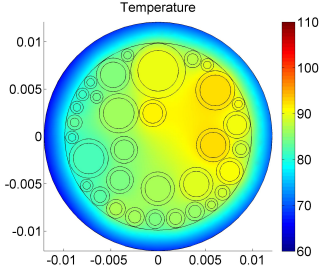

(a)

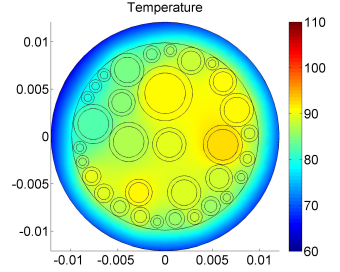

(b)

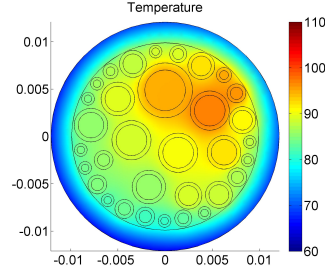

(c)

Fig. 20: Improved cable configurations obtained by application of the genetic algorithm for 33 single cables: (a) Best GA configuration with $J(\Omega)=92.43$, (b) second GA configuration with $J(\Omega)=93.81$, and (c) third GA configuration with $J(\Omega)=96.89$.

\begin{tabular}{cccccccccc}
\hline Generation & \multicolumn{7}{c}{ Individual No. } \\
No. & 1 & 2 & 3 & 4 & 5 & 6 & 7 & 8 & 9 \\
\hline 0 & 99.05 & 96.59 & 99.64 & 96.48 & 97.53 & 96.76 & 95.81 & 97.37 & 93.76 \\
1 & 93.76 & 95.39 & 96.30 & 99.12 & 93.09 & 95.68 & 92.61 & 95.13 & 98.45 \\
2 & 92.61 & 99.65 & 98.34 & 94.54 & 96.37 & 97.87 & 97.88 & 97.33 & 98.70 \\
3 & 92.61 & 96.90 & 97.92 & 92.43 & 96.00 & 95.86 & 94.92 & 95.04 & 98.16 \\
4 & 92.43 & 95.97 & 95.50 & 95.75 & 96.59 & 96.09 & 99.61 & 96.75 & 95.79 \\
5 & 92.43 & 98.18 & 98.11 & 93.81 & 97.45 & 99.12 & 98.65 & 97.04 & 96.89 \\
\hline
\end{tabular}

Table 7: Fitness values of all individuals for 6 generations in the progress of the genetic algorithm for multicables with 33 single cables.

of several single cables, even for limited space, is surprising in this context. In fact, this gradient based approach runs into local minima that are numerous for larger numbers of single cables. In combination with the genetic algorithm, the global optimum is however approximated in acceptable time, running several instances in parallel. 
We showed how a well thought-out and precise cable production process could improve the thermal on-board management in cars, just by varying the cable positions. Hardly any attention has been paid to this fact, yet. By now, the production process for multicables and cable harnesses is not as precise as necessary for an optimal design. The present works demonstrates that there exists a great potential to reduce temperatures in multicables with an optimized design.

\section{Acknowledgments}

The authors thank Prof. Thomas Apel, Benjamin Nolet and Max Winkler for their help in the implementation of the algorithms, for valuable hints concerning the problem definition resp. the optimization strategy and for proofreading of the article.

\section{References}

1. S.W. Churchill, R. Usagi. A general expression for the correlation of rates of transfer and other phenomena. AIChE J. 18(6) (1972), 1121-1128.

2. Ra. Ciegis, Re. Ciegis, M. Meilunas, G. Jankeviciute, V. Starikovtius. Parallel numerical algorithms for optimization of electrical cables. Math. Model. Anal. 13(4) (2008), 471482.

3. Ra. Ciegis, A. Ilgevicius, G. Jankeviciute, M. Meilunas. Determination of heat conductivity coefficient of a cable bundle by inverse problem solution method. Electron. Electr. Eng. 2 (2009), 77-80.

4. Ra. Ciegis, A. Ilgevicius, H.-D. Liess, M. Meilunas, O. Suboc. Numerical simulation of the heat conduction in electrical cables. Math. Model. Anal. 12 (2007), 425-439.

5. COMSOL Multiphysics. Reference guide version 3.5a. 2008.

6. M. Delfour, J.-P. Zolésio. Shapes and geometries. SIAM, Philadelphia, 2011.

7. P. Deuflhard. A modified Newton method for the solution of ill-conditioned systems of nonlinear equations with application to multiple shooting. Numer. Math. 22 (1974), $289-315$.

8. T.A. Davis. Algorithm 832: UMFPACK, an unsymmetric-pattern multifrontal method. ACM Transactions on Mathematical Software 30(2) (2004), 196-199.

9. T.A. Davis and I.S. Duff. An unsymmetric-pattern multifrontal method for sparse $L U$ factorization. SIAM Journal on Matrix Analysis and Applications 18(1) (1997), 140158.

10. K. Dvorsky. Analysis of a nonlinear boundary value problem with application to heat transfer in electric cables. Ph.D. dissertation, Fac. Aerosp. Tech., Univ. German Federal Armed Forces, Munich, 2013.

11. H. Harbrecht. On analytical derivatives for geometry optimization in the polarizable continuum model. J. Math. Chem. 49(9) (2011), 1928-1936.

12. H. Harbrecht, J. Tausch. An efficient numerical method for a shape-identification problem arising from the heat equation. Inverse Problems 27 (2011), 065013.

13. J. Haslinger, R.A.E. Mäkinen. Introduction to Shape Optimization: Theory, Approximation, and Computation. Advances in Design and Control, SIAM, Philadelphia, 2003.

14. R. Haupt, S. Haupt. Practical genetic algorithms. 2nd edition, John Wiley \& Sons, New York, NY, USA, 1998.

15. F. Hettlich, W. Rundell. The determination of a discontinuity in a conductivity from a single boundary measurement. Inverse Problems 14 (1998), 67-82.

16. F. Hettlich, W. Rundell. Identification of a discontinuous source in the heat equation. Inverse Problems 17 (2001), 1465-1482. 
17. S.Y. Huang, F. Mayinger. Wärmeübergang bei freier Konvektion um elliptische Rohre. Wärme- und Stoffübertragung 18(3) (1984), 175-183.

18. A. Ilgevicius. Analytical and numerical analysis and simulation of heat transfer in electrical conductors and fuses. Ph.D. dissertation, Fac. Elec. Inform. Tech., Univ. German Federal Armed Forces, Munich, 2004.

19. F. Loos. Joule Heating in Connecting Structures of Automotive Electric Devices Modelling, Simulation and Optimization. Ph.D. dissertation, Fac. Civ. Eng. Tech., Univ. German Federal Armed Forces, Munich, 2014.

20. F. Loos, K. Dvorsky, H.-D. Liess, Two approaches for heat transfer simulation of current carrying multicables. Math. Comp. Sim. 101(0) (2014), 13-30.

21. MathWorks. Using the genetic algorithm: user's guide (R2010b). Retrieved February 26, 2013 from www.mathworks.com/help/gads.

22. J. Murat, J. Simon. Étude de problèmes d'optimal design in Optimization Techniques, Modeling and Optimization in the Service of Man, edited by J. Céa, Lect. Notes Comput. Sci. 41, Springer-Verlag, Berlin, 54-62, 1976.

23. O. Pironneau. Optimal shape design for elliptic systems. Springer, New York, 1983.

24. O. Schenk and K. Gärtner. Solving unsymmetric sparse systems of linear equations with PARDISO. Journal of Future Generation Computer Systems 20(3) (2004), 475-487.

25. O. Schenk and K. Gärtner. On fast factorization pivoting methods for symmetric indefinite systems. Elec. Trans. Numer. Anal. 23 (2006), 158-179.

26. S. Schmidt. Efficient large scale aerodynamic design based on shape calculus. Ph.D. dissertation, University of Trier, Germany, 2010.

27. J. Sokolowski, J.-P. Zolésio. Introduction to shape optimization. Springer, Berlin, 1992.

28. A. Wächter. Short tutorial: Getting started with IPOPT in 90 minutes. In Combinatorial Scientific Computing, Dagstuhl Seminar Proceedings 09061, Schloss Dagstuhl - Leibniz-Zentrum für Informatik, Dagstuhl, Germany, 2009, http://drops.dagstuhl.de/opus/volltexte/2009/2089.

29. A. Wächter and L.T. Biegler. Line search filter methods for nonlinear programming: Motivation and global convergence. SIAM Journal on Optimization 16(1) (2005), 1-31.

30. A. Wächter and L.T. Biegler. On the implementation of a primal-dual interior point filter line search algorithm for large-scale nonlinear programming. Mathematical Programming 106(1) (2006), 25-57. 